\title{
New constraints on the disk characteristics and companion candidates around T Chamaeleontis with VLT/SPHERE`
}

\author{
A. Pohl ${ }^{1,2, \star \star}$, E. Sissa ${ }^{3,4}$, M. Langlois ${ }^{5,6}$, A. Müller ${ }^{1,7}$, C. Ginski ${ }^{8,9}$, R. G. van Holstein ${ }^{8}$, A. Vigan ${ }^{6}$, D. Mesa ${ }^{3}$, \\ A.-L. Maire ${ }^{1}$, Th. Henning ${ }^{1}$, R. Gratton ${ }^{3}$, J. Olofsson ${ }^{10,1,11}$, R. van Boekel ${ }^{1}$, M. Benisty ${ }^{12}$, B. Biller ${ }^{1,13}$, \\ A. Boccaletti ${ }^{14}$, G. Chauvin ${ }^{12}$, S. Daemgen ${ }^{15}$, J. de Boer ${ }^{8}$, S. Desidera ${ }^{3}$, C. Dominik ${ }^{9}$, A. Garufi ${ }^{15}$, M. Janson ${ }^{1,16}$, \\ Q. Kral ${ }^{17}$, F. Ménard ${ }^{12}$, C. Pinte $^{12}$, T. Stolker ${ }^{9}$, J. Szulágyi ${ }^{15}$, A. Zurlo ${ }^{6,18,19}$, M. Bonnefoy ${ }^{12}$, A. Cheetham ${ }^{20}$, \\ M. Cudel ${ }^{12}$, M. Feldt ${ }^{1}$, M. Kasper ${ }^{21}$, A.-M. Lagrange ${ }^{12}$, C. Perrot $^{14}$, and F. Wildi ${ }^{20}$
}

(Affiliations can be found after the references)

Received 12 December 2016/ Accepted 9 May 2017

\begin{abstract}
Context. The transition disk around the $\mathrm{T}$ Tauri star $\mathrm{T}$ Cha possesses a large gap, making it a prime target for high-resolution imaging in the context of planet formation.

Aims. We aim to find signs of disk evolutionary processes by studying the disk geometry and the dust grain properties at its surface, and to search for companion candidates.

Methods. We analyze a set of VLT/SPHERE data at near-infrared and optical wavelengths. We performed polarimetric imaging of T Cha with IRDIS $(1.6 \mu \mathrm{m})$ and ZIMPOL $(0.5-0.9 \mu \mathrm{m})$, and obtained intensity images from IRDIS dual-band imaging with simultaneous spectro-imaging with IFS $(0.9-1.3 \mu \mathrm{m})$.

Results. The disk around $\mathrm{T}$ Cha is detected in all observing modes and its outer disk is resolved in scattered light with unprecedented angular resolution and signal-to-noise. The images reveal a highly inclined disk with a noticeable east-west brightness asymmetry. The significant amount of non-azimuthal polarization signal in the $U_{\phi}$ images, with a $U_{\phi} / Q_{\phi}$ peak-to-peak value of $14 \%$, is in accordance with theoretical studies on multiple scattering in an inclined disk. Our optimal axisymmetric radiative transfer model considers two coplanar inner and outer disks, separated by a gap of $0 \prime 228(\sim 30 \mathrm{au})$ in size, which is larger than previously thought. We derive a disk inclination of $\sim 69 \mathrm{deg}$ and PA of $\sim 114 \mathrm{deg}$. In order to self-consistently reproduce the intensity and polarimetric images, the dust grains, responsible for the scattered light, need to be dominated by sizes of around ten microns. A point source is detected at an angular distance of 3.5" from the central star. It is, however, found not to be co-moving. Conclusions. We confirm that the dominant source of emission is forward scattered light from the near edge of the outer disk. Our point source analysis rules out the presence of a companion with mass larger than $\sim 8.5 M_{\text {jup }}$ between $0{ }^{\prime \prime} 1$ and $0 ! 3$. The detection limit decreases to $\sim 2 M_{\text {jup }}$ for 0,3 to $4.0^{\prime \prime}$.
\end{abstract}

Key words. stars: individual: T Cha - protoplanetary disks - techniques: polarimetric - radiative transfer - scattering - circumstellar matter

\section{Introduction}

Recently developed high-resolution and high-contrast imaging instruments provide the excellent capability to directly obtain images of protoplanetary disks in scattered light and thermal emission. Protoplanetary disks are optically thick in the optical and near-infrared (NIR), so that scattered light imaging probes (sub-)micron-sized dust grains in the disk surface layer. Contrarily, (sub-)mm observations trace larger, mm-sized grains located in the disk midplane. The detection of (non-)axisymmetric disk features is fundamental in improving our current understanding of disk evolution and planet formation. Transition disks with large gas and dust gaps (see e.g. mid-infrared surveys by Brown et al. 2007; Merín et al. 2010; van der Marel et al. 2016 and $\mathrm{mm}$ observations by Isella et al. 2010a,b; Andrews et al. 2011; van der Marel et al. 2015) are particularly interesting targets, since they host possible planet-forming hotspots and may show signposts of planet-disk interaction processes. In recent observational studies, giant gaps and cavities have been directly

^ Based on observations made with European Southern Observatory (ESO) telescopes at the Paranal Observatory in Chile, under program IDs 095.C-0298(B), 096.C-0248(B) and 096.C-0248(C).

$\star \star$ Corresponding author: A. Pohl, e-mail: pohl@mpia.de imaged in scattered light observations of transition disk systems (e.g., Thalmann et al. 2010, 2015; Hashimoto et al. 2012; Avenhaus et al. 2014; Follette et al. 2015; Ohta et al. 2016; Stolker et al. 2016; Benisty et al. 2017).

T Chamaeleontis (T Cha) is a $\sim 2-12$ Myr old (Brown et al. 2007; Torres et al. 2008; Murphy et al. 2013) T Tauri star (spectral type G8, Alcala et al. 1993) at an estimated distance of $107 \pm 3$ pc (first Gaia data release, Gaia Collaboration 2016) surrounded by a transition disk. Several recent studies covering a wide wavelength range dealt with constraining the disk geometry around T Cha. Brown et al. (2007) studied its spectral energy distribution (SED), which features a significant deficit of mid-infrared (MIR) emission. With radiative transfer based SED fitting they modeled this deficit by introducing a gap between 0.2 and $15 \mathrm{au}$, dividing the disk into two spatially separated parts. Hence, it became a prime candidate for investigating signatures of ongoing planet formation. Olofsson et al. (2011) presented spatially resolved, interferometric observations at high angular resolution in the NIR from the AMBER instrument at the Very Large Telescope Interferometer (VLTI) to study the inner disk's structure. The inner disk is found to be extremely narrow and located close to the star with an extension from 0.13 
to $0.17 \mathrm{au}$. Olofsson et al. (2013) presented a radiative transfer model accounting for several further interferometric and photometric observations, including VLTI/PIONIER, VLTI/MIDI and NACO/Sparse Aperture Masking (SAM) data, which further constrains the inner disk to extend from 0.07 to 0.11 au. Further SED modeling of T Cha by Cieza et al. (2011) suggests that there is a high degeneracy especially for the outer disk geometry, since a very compact outer disk provides an equally good fit to the Herschel data as a much larger, but tenuous disk with a very steep surface density profile. High-resolution Atacama Large Millimeter/sub-millimeter Array (ALMA) observations of the $850 \mu \mathrm{m}$ dust continuum as well as of several emission lines presented by Huélamo et al. (2015) spatially resolved the outer disk around $\mathrm{T}$ Cha and helped to break the degeneracy of previous outer disk models. They report a compact dusty disk, where the continuum intensity profile displays two emission bumps separated by $40 \mathrm{au}$, indicating an inner gap radius of $20 \mathrm{au}$ and an outer disk radius of $\sim 80 \mathrm{au}$. In contrast, the gaseous disk is larger by almost a factor of three, giving a radius of $\sim 230$ au based on the detection of $\mathrm{CO}(3-2)$ molecular emission. Huélamo et al. (2015) derived a disk inclination (incl) of $67^{\circ} \pm 5^{\circ}$ and a position angle (PA) of $113^{\circ} \pm 6^{\circ}$ by fitting a Gaussian to the $\mathrm{CO}(3-2)$ integrated emission map.

All previous observations clearly confirm that there must be a significant gap in the disk dust density distribution, while its origin is still debated. In general, radial gap structures can be created by a number of processes, including grain growth (e.g., Dullemond \& Dominik 2005), effects of the magnetorotational instability (MRI) at the outer edge of a dead-zone (e.g., Flock et al. 2015), a close (sub-)stellar companion or the dynamical interaction of a planet formed within the disk (e.g., Rice et al. 2003). For the latter, the disk density modification results from the torques exerted on the disk by the planet and by the disk itself. The planet pushes away the surrounding material, the outer part of the disk outward and the inner part inward, thereby opening a gap (e.g., Lin \& Papaloizou 1979; Crida et al. 2006). Studies of observational signatures of planet-disk interaction processes based on numerical simulations suggest that gaps detected in scattered light may be opened by planets (e.g., Pinilla et al. 2015; Dong et al. 2015, 2016; Juhász et al. 2015; Pohl et al. 2015). Using NACO/SAM Huélamo et al. (2011) detected a companion candidate at a projected distance of 6.7 au from the primary, which is well within the previously described disk gap. However, an analysis of several L' and $K_{\mathrm{s}}$ data sets covering a period of three years ruled out this companion hypothesis (Olofsson et al. 2013; Cheetham et al. 2015). The absence of relative motion for the companion candidate favors a stationary structure consistent with scattered light from a highly inclined disk. Sallum et al. (2015) checked if the closure phase signal from their VLT/NACO and Magellan/MagAO/Clio2 data shows any variation in time, which is not expected for the disk scattering model. While NACO L' data from 2011 and 2013 support the hypothesis of constant scattered light from the disk, the best fits for two other NACO data sets are inconsistent, requiring temporal variability in the amount of scattered light. Apart from this variability argument, Sallum et al. (2015) showed by means of Monte Carlo simulations that noise fluctuations could also cause the changing structure in the NACO and MagAO reconstructed images.

In this work we present the first scattered light observations of T Cha obtained with the SPHERE instrument (SpectroPolarimeter High contrast Exoplanet REsearch, Beuzit et al. 2008) at the Very Large Telescope (VLT). The target is now one of the few T Tauri stars to have been spatially resolved in high detail in scattered light. We used Polarimetric Differential Imaging (PDI) complemented with total intensity images obtained with the angular differential imaging (ADI) technique. The observations provide the first spatially resolved high-contrast images of T Cha in the optical and NIR. Our focus is set on analyzing the scattered light properties of the disk. We perform physical modeling of the disk via radiative transfer calculations, which helps us to further constrain the disk's geometry and grain size distribution. Images in the full Stokes vector are calculated in order to consistently reproduce the observed total and polarized intensity. Furthermore, the total intensity images are used for a detailed search for substellar companion candidates and, in case of non-detection, to place constraints on the mass of putative companions using the detection limits. This paper is laid out as follows. In Sect. 2 we describe our observations and the data reduction procedures; their results are shown in Sect. 3. Section 4 presents our results of the radiative transfer disk modeling and the search for substellar companions. A detailed discussion in Sect. 5 follows. In Sect. 6 we summarize the main conclusions of this work.

\section{SPHERE observations and data reduction}

Observations of $\mathrm{T}$ Cha were performed during the nights of 30 May 2015, 19 February 2016, and 31 March 2016 with several sub-systems of the high-contrast imager SPHERE equipped with an extreme adaptive optics system (SAXO, Fusco et al. 2006, 2014) and mounted on the VLT at Cerro Paranal, Chile. All observations were part of the SPHERE consortium guaranteed time program under IDs 095.C-0298(B) and 096.C0248(B/C). The Infra-Red Dual-beam Imager and Spectrograph (IRDIS, Dohlen et al. 2008) and the Zurich IMaging POLarimeter (ZIMPOL, Thalmann et al. 2008; Schmid et al. 2012) were used in Dual-band Polarimetric Imaging (DPI) mode (Langlois et al. 2014) and in field stabilized (P2) mode, respectively. In addition, data were taken simultaneously with IRDIS in Dual-Band Imaging (DBI; Vigan et al. 2010) mode and the Integral Field Spectrograph (IFS, Claudi et al. 2008). In this IRDIFS mode, IRDIS is operated in the filter pair $H 2 H 3(1.593 \mu \mathrm{m}$ and $1.667 \mu \mathrm{m})$ and IFS in $Y J(0.95-1.35 \mu \mathrm{m})$ mode. Table 1 summarizes the observations and instrumental setups for each instrument. The Strehl ratio estimation (provided by SPARTA files) is based on an extrapolation of the phase variance deduced from the reconstruction of SAXO open-loop data using a deformable mirror, tip-tilt voltages, and wavefront sensor closed-loop data (Fusco et al. 2004). The observing conditions and the different data reduction methods for each data set taken by the various sub-systems are described in detail in Sects. 2.1-2.3.

\subsection{IRDIS-DPI (H-band)}

The IRDIS-DPI observations of $\mathrm{T}$ Cha were carried out on 19 February 2016 with the $B B_{-} H$ filter $\left(\lambda_{\mathrm{c}}=1.625 \mu \mathrm{m}\right)$ using an apodized pupil Lyot coronagraph with a mask diameter of 185 mas (Soummer 2005; Boccaletti et al. 2008). Dark and flat field calibration were obtained during the following day. Thirty polarimetric cycles were taken, consisting of one data cube for each of the four half wave plate (HWP) positions $\left(0^{\circ}, 45^{\circ}, 22.5^{\circ}\right.$ and $\left.67.5^{\circ}\right)$. Dedicated coronagraphic images were taken at the beginning and at the end of the science sequence to determine accurately the position of the star behind the coronagraph. For this calibration a periodic amplitude is applied to the deformable mirror, which produces four equidistant, crosswise satellite spots 
Table 1. Overview of observational data sets.

\begin{tabular}{ccccccccc}
\hline \hline Date & Instrument & Mode & Filter & DIT [s] $\times$ NDIT & PC & $t_{\text {tot }}[\mathrm{min}]$ & Seeing ["] & $H$-band Strehl [\%] \\
\hline 2015 May 30 & IRDIS & IRDIFS & $D B \_H 2 H 3$ & $64 \times 96$ & - & 102 & $0.5-0.85$ & $27 \pm 13$ \\
2015 May 30 & IFS & IRDIFS & $Y J$ & $64 \times 96$ & - & 102 & $0.5-0.85$ & $27 \pm 13$ \\
2016 February 19 & IRDIS & DPI & $H$ & $32 \times 1$ & 30 & 64 & $0.9-1.0$ & $76 \pm 4$ \\
2016 March 31 & ZIMPOL & P2 & $V B B$ & $40 \times 2$ & 18 & 96 & $0.8-1.1$ & $87 \pm 3$ \\
\hline
\end{tabular}

Notes. Both filters of ZIMPOL were set to the Very Broad Band $(V B B)$ filter covering a wide wavelength regime from $R$ - to $I$-band. The following coronagraphs were used: N_ALC_YJH_SDIT for IRDIS/IFS and V_CLC_S_WF for ZIMPOL. DIT stands for the detector integration time and NDIT corresponds to the number of frames in the sequence. PC indicates the number of polarimetric cycles. The Strehl is calculated for the $H$-band. This leads to a Strehl of $\sim 43 \%$ for the ZIMPOL data at $0.65 \mu \mathrm{m}$.

of the stellar PSF outside of the coronagraph. The data were reduced following the prescriptions of Avenhaus et al. (2014) and Ginski et al. (2016), who consider the radial Stokes formalism. The first step consists of standard calibration routines, including dark-frame subtraction, flat-fielding and bad-pixel correction. These images are split into two individual frames representing the left and right sides (parallel and perpendicular polarized beams, respectively), and the precise position of the central star is measured using the star center calibration frames on both image sides separately. Then, the right side of the image is shifted and subtracted from the left side. To obtain clean Stokes $Q$ and $U$ images, that is, to correct for instrumental polarization downstream of the HWP's position in the optical path, $Q^{+}$and $Q^{-}$ $\left(0^{\circ}\right.$ and $\left.45^{\circ}\right)$, and $U^{+}$and $U^{-}\left(22.5^{\circ}\right.$ and $\left.67.5^{\circ}\right)$ are subtracted, respectively. However, there might be still an instrumental polarization left upstream of the HWP in the final $Q$ and $U$ images, which is assumed to be proportional to the total intensity image as shown in Canovas et al. (2011). To obtain this residual instrumental signal, the azimuthal Stokes components are computed from (cf. Schmid et al. 2006)

$Q_{\phi}=+Q \cos 2 \phi+U \sin 2 \phi$,

$U_{\phi}=-Q \sin 2 \phi+U \cos 2 \phi$.

The azimuth $\phi$ is defined with respect to the stellar position $\left(x_{0}, y_{0}\right)$ as

$\phi=\arctan \frac{x-x_{0}}{y-y_{0}}$.

As shown by Canovas et al. (2015), the signal in the $U_{\phi}$ frame should be small for a centrally illuminated symmetrical disk. We thus determined the scaling factor for our second instrumental polarization correction such that the (absolute) signal in an annulus around the central star in the $U_{\phi}$ frame is minimized. We then subtract the scaled Stokes $I$ frame from the $Q$ and $U$ frame and use these final corrected frames to create the $Q_{\phi}$ and $U_{\phi}$ images displayed in Fig. 1.

To cross-check the IRDIS-DPI results, and especially to test the reliability of the $U_{\phi}$ minimization technique in the context of an inclined disk, we additionally perform an alternative reduction procedure. This includes a proper polarimetric calibration using a Mueller matrix model, whose details will be presented in van Holstein et al. (in prep.) and de Boer et al. (in prep.). A short explanation of the method and the corresponding results for T Cha can be found in Sect. 3.1 and Fig. 2.

\subsection{ZIMPOL P2 (VBB filter)}

T Cha was observed during the night of 31 March 2016 with the SlowPolarimetry detector mode of ZIMPOL using the very broad band filter $(V B B)$. The $V B B$ filter covers a wide wavelength range from $R$ - to $I$-band $(0.55-0.87 \mu \mathrm{m})$. These observations were also obtained with an apodized Lyot coronagraph (mask diameter of $\sim 185$ mas).

The ZIMPOL data were reduced following mostly the same strategy as described for the IRDIS data in the previous section. The main difference between the two data sets is the different structure of the ZIMPOL data. In ZIMPOL the two perpendicular polarization directions for each HWP position are recorded quasi-simultaneously on the same detector pixels. For a more detailed description of the instrument and the specialized data reduction steps involved we refer to Thalmann et al. (2008) and Schmid et al. (2012). We process both ZIMPOL detector images independently and only combine the images after the final data reduction to increase the $\mathrm{S} / \mathrm{N}$. We first bias subtract and flat field the individual frames. We then extract the two perpendicular polarization directions from the interlaced rows in each frame, resulting in two $1024 \times 512$ pixel images per original frame. We then correct for charge-shifting artifacts by always combining two consecutive frames of the observation sequence. To create quadratic images we then bin each image by a factor of two along the $x$-axis. Finally we subtract the two perpendicular polarization directions from each other to get $Q^{+}, Q^{-}, U^{+}$and $U^{-}$ frames (depending on the HWP position). These are then combined to create the final $Q$ and $U$ frames identical to the IRDIS reduction. In a last step we again calculate the azimuthal Stokes components $Q_{\phi}$ and $U_{\phi}$ and employ the instrumental polarization correction from Canovas et al. (2011). The resulting images (after combination of both ZIMPOL images) are also displayed in Fig. 1.

\subsection{IRDIS-DBI (H2H3-bands) and IFS (YJ-band)}

T Cha IRDIFS observations where obtained during the night of 30 May 2015 as part of the SpHere INfrared survey for Exoplanets (SHINE; Chauvin et al., in prep.) using the SHINE standard setup: pupil-stabilized images with IFS operating in $Y J$ mode ( 39 channels between 0.95 and $1.35 \mu \mathrm{m}$ ) and IRDIS working in DBI mode using the $H 2 H 3$ filter pair $\left(\lambda_{\mathrm{H} 2}=1.593 \mu \mathrm{m}\right.$; $\left.\lambda_{\mathrm{H} 3}=1.667 \mu \mathrm{m}\right)$. This observing strategy allows for performing ADI (Marois et al. 2006) in order to reach high contrast. The spectral resolution of IFS $Y J$ data amounts to $R \sim 50$. The observations lasted about $6100 \mathrm{~s}$ with a field rotation of $\sim 28^{\circ}$. Since the target is located far to the south, obtaining a good rotation is challenging. The unstable weather conditions (Differential Image Motion Monitor (DIMM) seeing varied from 0 0.5 to 0 ".85 and clouds passing by) caused flux variations of up to one order of magnitude during the sequence. 


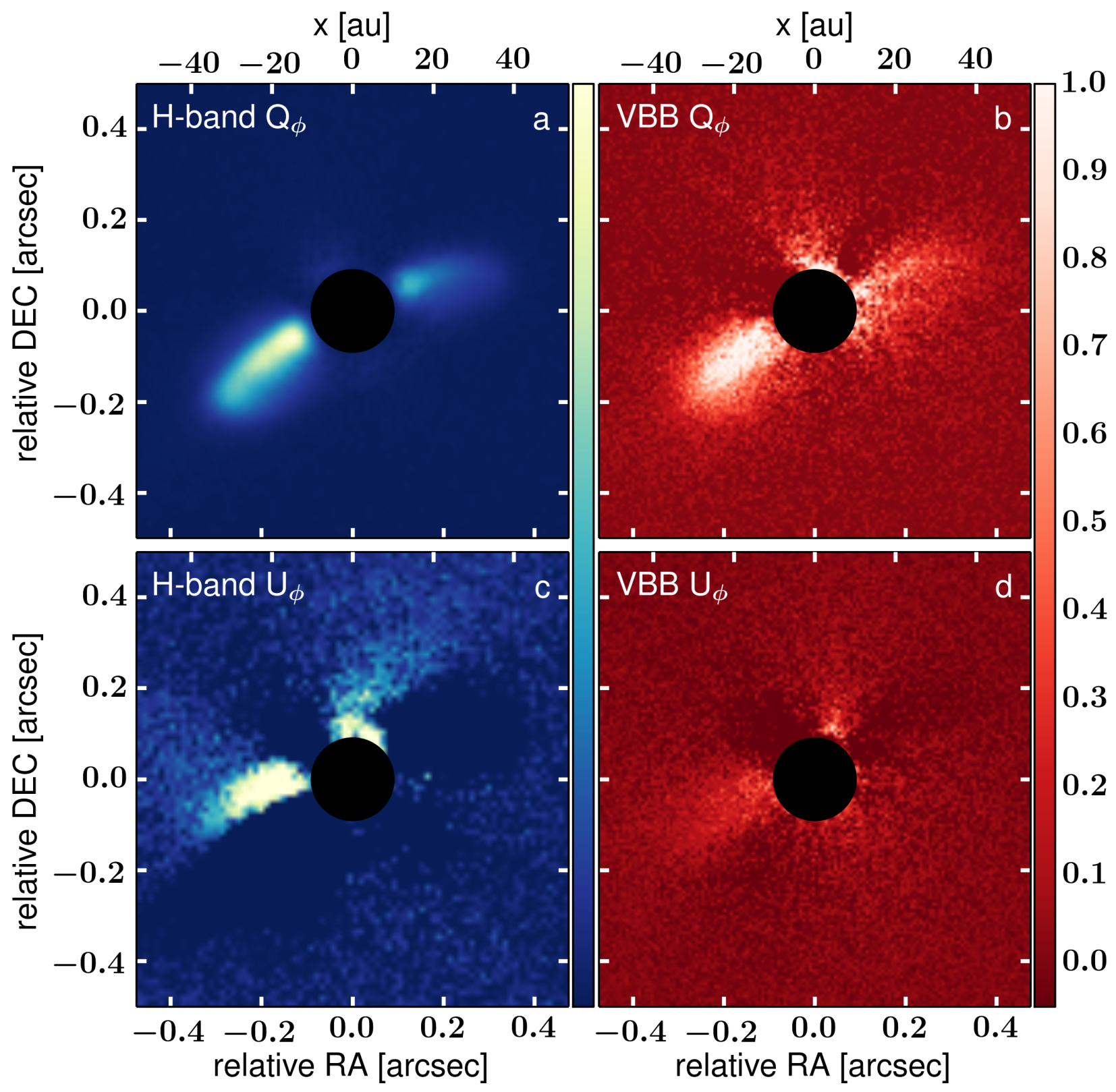

Fig. 1. IRDIS-DPI $H$-band and ZIMPOL P2 $V B B$-filter $Q_{\phi}$ (top row) and $U_{\phi}$ (bottom row) images. North is up, east is left. All images are normalized to the highest disk brightness. The dynamical range for the color scaling is the same for the two images of the top (1000) and bottom row (20), respectively. An apodized Lyot coronagraph with a mask diameter of $\sim 185$ mas was used. The inner 0 "'18 are masked, represented by the black circular area. Negative values of $U_{\phi}$ are saturated at dark blue and dark red color, respectively.

The basic steps of the first IRDIS data reduction consist of flat-field and bad-pixel correction, cosmic ray detection and correction, and sky subtraction. Because of the variable atmospheric conditions during the observations, a very strict frame selection is applied at the end of the basic reduction and eventually only 42 out of 96 frames were used. This corresponds to selecting frames with Strehl ratio larger than $\sim 25 \%$. The modeling and subtraction of the speckles follows the MPIA-PCA pipeline from André Müller. This is based on a principal component analysis (PCA) after Absil et al. (2013), which uses the Karhunen-Loéve Image Projection (KLIP) algorithm from Soummer et al. (2012). We apply the following basic reduction steps: (1) Gaussian smoothing with half of the estimated FWHM; (2) normalization of the images based on the measured peak flux of the PSF images; (3) PCA and subtraction of the modeled noise; and (4) derotation and averaging of the images. Each frame is divided into annuli of the size of the estimated FWHM. The noise of a single frame and annulus is modeled by PCA using a fixed value of five modes, which is found to be the best value after several attempts. In addition, only frames for modeling the noise of the individual frame were selected where a minimum protection angle can be guaranteed. This means that with this adaptive approach the effect of self subtraction for an extended source is minimized and the $\mathrm{S} / \mathrm{N}$ maximized.

To cross-check the IRDIS-DBI results, we perform a parallel reduction using the SPHERE Data Reduction and Handling pipeline (DRH, Pavlov et al. 2008) implemented at the SPHERE Data Center. This includes dark and sky subtraction, bad-pixel removal, flat-field correction, anamorphism correction, and wavelength calibration. After these first steps the data were sorted according to their quality. Because of the difficult observing conditions, we use stringent frame selection 
(using 77 frames out of 96). This roughly corresponds to selecting frames with Strehl ratio greater than $15 \%$ and leads to an average $H$-band Strehl of $\sim 33 \%$. The location of the star is identified with the four symmetrical satellite spots generated from a waffle pattern on the deformable mirror. Then, to remove the stellar halo and to achieve high-contrast, the data were processed with the SpeCal pipeline developed for the SHINE survey (Galicher et al., in prep.); this implements a variety of ADI-based algorithms: Classical Angular Differential Imaging (cADI, Marois et al. 2006), Template Locally Optimized Combination of Images (TLOCI, Marois et al. 2006) and PCA (Soummer et al. 2012; Amara \& Quanz 2012). In the following we discuss the results based on the TLOCI, and PCA images for the morphology and photometric analyses. Separate reductions were performed for the extraction of the disk. In particular, for the SpeCal PCA reduction a small number of PCA modes is used in order to enable optimal retrieval of the disk. The reduced number of modes, between two and four, are determined by maximizing the SNR inside a region delimited by the disk location. For the contrast curves, TLOCI images were considered because they provide the best compromise of contrast, stellar rejection, and throughput correction for the point source detection.

The data reduction for the IFS is performed using tools available at the SPHERE Data Center at IPAG following the procedure described in Mesa et al. (2015) and in Zurlo et al. (2014). Using the SPHERE DRH software we apply the appropriate calibrations (dark, flat, spectral positions, wavelength calibration and instrument flat) to create a calibrated datacube composed of 39 images of different wavelengths for each frame obtained during the observations. Similar to the procedure used for IRDIS, in order to take into account the very variable weather conditions, we apply a frame selection resulting in 76 frames out of the original 96. A frame is considered as "bad" if the adaptive optics loop opens or the star exits the coronagraph region, causing an excess of light in the central part of the image. For each frame two central areas with 20 and 160 pixels per side are defined, for which the flux ratio is determined. Frames are rejected by an automated sorting if this ratio exceeds $130 \%$ of the median value. The position of the star behind the coronagraph is estimated from images with four satellite spots, symmetric with respect to the central star taken just before and after the standard coronagraphic observations. Exploiting these images we are then able to define the re-scaling factor for images at different wavelengths to maintain the speckle pattern as stable as possible. Moreover, we are able to combine those images using the PCA algorithm from Soummer et al. (2012) to implement both ADI and Spectral Differential Imaging (SDI, Racine et al. 1999) in order to remove the speckle noise.

\section{Results}

The disk of $\mathrm{T}$ Cha is detected in all data sets presented in this study. The analysis of the disk geometry primarily focuses on the IRDIS-DPI and IRDIS-ADI images (see Sect. 4.1). Furthermore, the ADI images are used to search for point-source signals focusing on non-polarized companions, because this data set reaches a higher contrast (Sects. 4.2 and 4.3).

\subsection{Polarized intensity images}

Figure 1 shows the reduced $Q_{\phi}$ and $U_{\phi}$ images of the IRDIS-DPI $H$-band and ZIMPOL $V B B$ observations of T Cha described in Sects. 2.1 and 2.2. The dark central region corresponds to the area masked by the coronagraph. The disk is clearly detected in the IRDIS $Q_{\phi}$ image, which gives by far the best quality view of the outer disk structure and its rim in scattered light for $\mathrm{T}$ Cha. Our SPHERE observations support a high disk inclination with respect to the line of sight, in agreement with the model by Huélamo et al. (2015). Scattered light is detected out to a projected distance of $\sim 0^{\prime \prime} .39$ ( $\sim 42 \mathrm{au}$ ) from the central star concentrated in a bright arc with, however, a significant difference in brightness between the east and west sides (factor of $\sim 2.5$ ). This is further discussed in Sects. 4.1.2 and 5.3. In the reduced $U_{\phi}$ image there is some residual signal left, which has usually been interpreted as instrumental effects or as imperfect centering of the images. However, since the $U_{\phi} / Q_{\phi}$ peak-to-peak value amounts to $9 \%$ and owing to the high inclination of the disk around T Cha (69 deg is determined from the total intensity image modeling, see Sect. 4.1.2), multiple scattering (e.g., Bastien \& Menard 1990; Fischer et al. 1996; Ageorges et al. 1996), that is, scattering of already polarized light, in the inner disk might be the prime contributor to this signal. This is consistent with a theoretical study by Canovas et al. (2015), who found that even for moderate disk inclinations multiple scattering alone can produce significant non-azimuthal polarization above the noise level in the $U_{\phi}$ images. They showed that the $U_{\phi} / Q_{\phi}$ peak-to-peak value can even go up to $50 \%$ for a disk inclination of 70 deg depending on the mass and grain size distribution of the disk model. We note that the exact geometrical structure of the $U_{\phi}$ signal might be influenced by the reduction method described in Sect. 2.1 (correction for the instrumental crosstalk by minimizing $U_{\phi}$ ). Therefore, we are not going to force our model to also fit the $U_{\phi}$ in addition to the $Q_{\phi}$. However, in order to prove that the $U_{\phi}$ signal is indeed real, we evaluate our IRDIS-DPI data with a newly developed reduction method, independent of the one presented in Sect. 2.1. By using the detailed Mueller matrix model of van Holstein et al. (in prep.) and de Boer et al. (in prep.) we correct our measurements for instrumental polarization effects. This model describes the complete optical path of SPHERE/IRDIS, i.e. telescope and instrument, and has been fully validated with measurements using SPHERE's internal source and observations of unpolarized standard stars (van Holstein et al., in prep.). The images of Stokes $Q$ and $U$ incident on the telescope are computed by setting up a system of equations describing every measurement of $Q$ and $U$ and solving it - for every pixel individually - using linear least-squares. The resulting $Q_{\phi}$ image (Fig. 2, left panel) is very similar to the one from the first reduction (Fig. 1, top left panel). The new $U_{\phi}$ image (Fig. 2, middle panel) has a higher accuracy than the one from the reduction that minimizes $U_{\phi}$ (Fig. 1, bottom left panel), in particular because no assumptions about the angle of linear polarization of the source are made to correct for the instrumental polarization. The $U_{\phi}$ image is cleaner and shows more symmetry in the sense that there is also a strong signal to the south-west. On the top right, the positive $U_{\phi}$ signal from Fig. 1, bottom left, is not visible anymore. The right panel of Fig. 2 shows the polarized intensity overplotted with polarization vectors representing the angle of linear polarization. This strengthens that there is a clear departure from azimuthal polarization. For the model-corrected images the $U_{\phi} / Q_{\phi}$ peak-to-peak value increases from $9 \%$ to $14 \%$, suggesting that some of the actual physical $U_{\phi}$ signal has been removed in the original reduction method due to the $U_{\phi}$ minimization procedure. A detailed comparison between different reduction methods and the specific influence on the left-over $U_{\phi}$ signal will be the topic of the two follow-up SPHERE papers.

The optical images obtained with ZIMPOL (Fig. 1, right panel) corroborate the disk geometry, but the bad weather conditions and low Strehl ratio $(43 \%$ at $0.65 \mu \mathrm{m})$ of this observation 


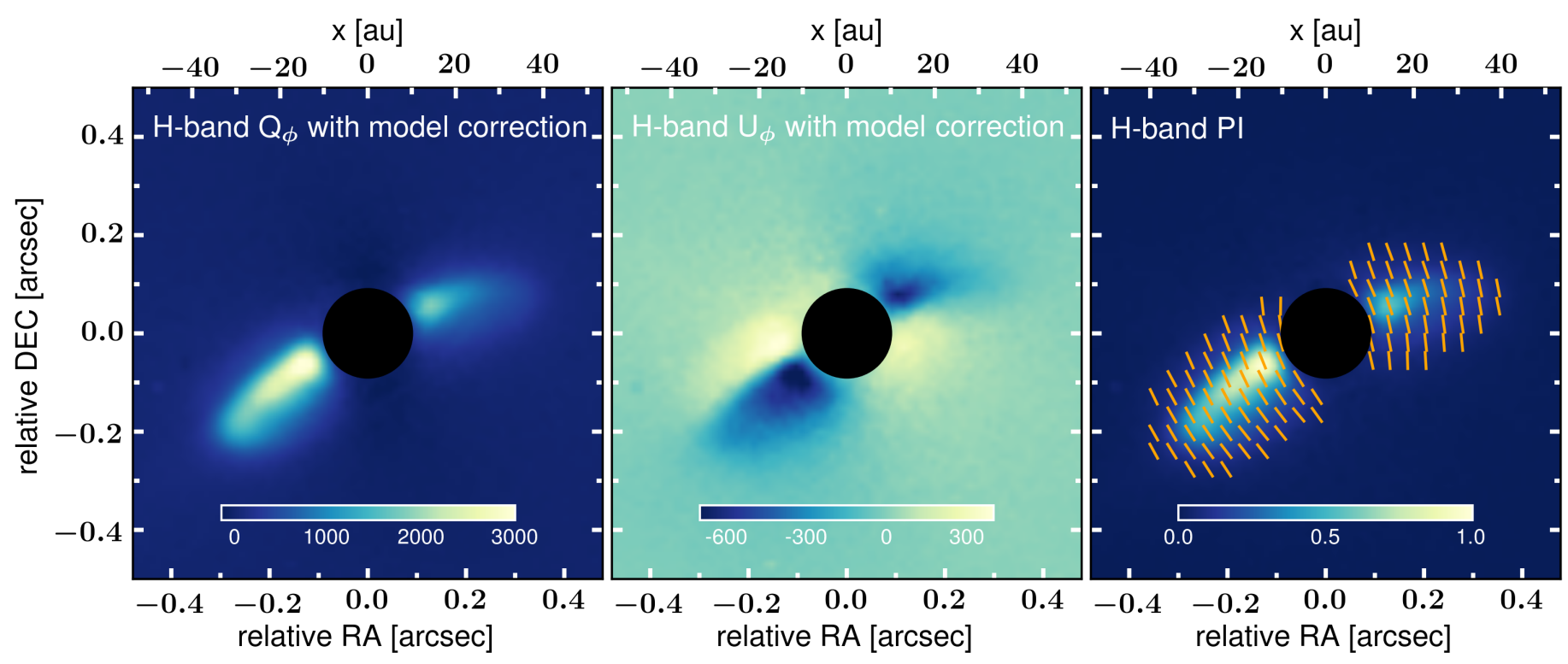

Fig. 2. Mueller matrix model-corrected IRDIS-DPI $Q_{\phi}(l e f t), U_{\phi}$ (middle) and polarized intensity $P I=\sqrt{Q^{2}+U^{2}}$ (right) images. North is up, east is left. Note that the $Q_{\phi}$ and $U_{\phi}$ images are not normalized/saturated here on purpose to emphasize their partially negative signal. The inner 0 "'.18 are masked, represented by the black circular area. The orange stripes in the right image represent the angle of linear polarization (fixed length, not scaled with the degree of polarization).

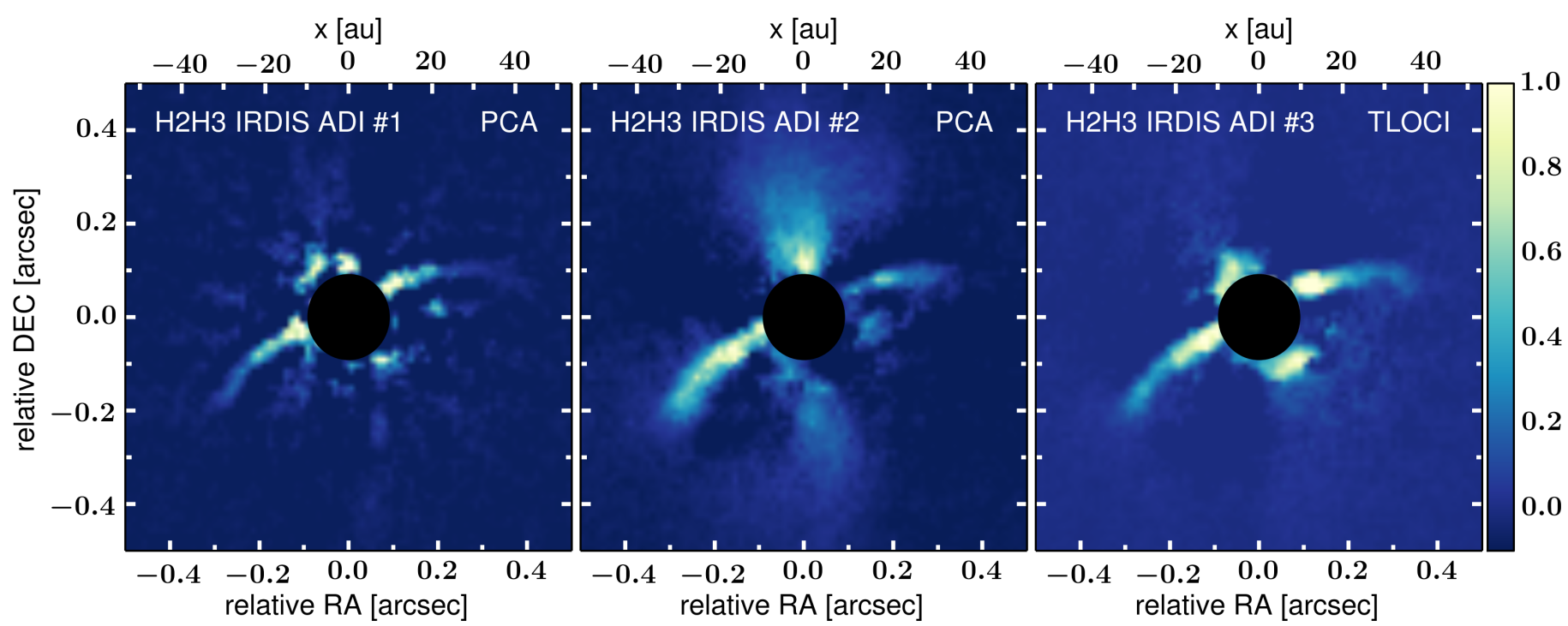

Fig. 3. IRDIS-ADI H2H3-band images (mean across the wavelengths) based on three different reduction pipelines (\#1: MPIA-PCA, \#2: SpeCalPCA and \#3: SpeCal-TLOCI). North is up, east is left. The images are normalized to the highest disk brightness and the color scales consider the same dynamical range. The inner 0 "'18 region masked by the coronagraph is represented by the black circular area.

lead to a rather blurred structure. Again, positive and negative patterns (dark and bright color) alternate in the $U_{\phi}$ image, where these negative patterns are practically at the same location as in the IRDIS $U_{\phi}$ image.

\subsection{Total intensity images}

In addition to the polarimetric images, the IRDIS-ADI $\mathrm{H}_{2} \mathrm{H3}$ intensity images in Fig. 3 also clearly show the inclined disk around $\mathrm{T}$ Cha. It even more strongly brings out the inner rim of the outer disk on the far side, visible as a faint arc below the coronagraph. The double-arch structure is a recurrent new form of features we have been detecting with high-contrast imaging instruments such as SPHERE (cf. Janson et al. 2016; Garufi et al. 2016). We note that because of the ADI processing this image may have been biased and is not a faithful representation of the true intensity and geometry. The residual signal northeast of the image center is likely due to stellar residuals. However, this signal is almost aligned with the near minor axis, so the possibility that it is real cannot be completely excluded. In fact, it could be the marginal detection of some material outward of the ring with high scattering efficiency. The brightness asymmetry between the west and east disk wings is as pronounced as in the polarimetric images, especially for reduction \#2. Figure 4 illustrates the median IFS image across the wavelength range from $Y$ - to $J$-band. The disk is nicely resolved, confirming the disk geometry and surface brightness extension stated above. 


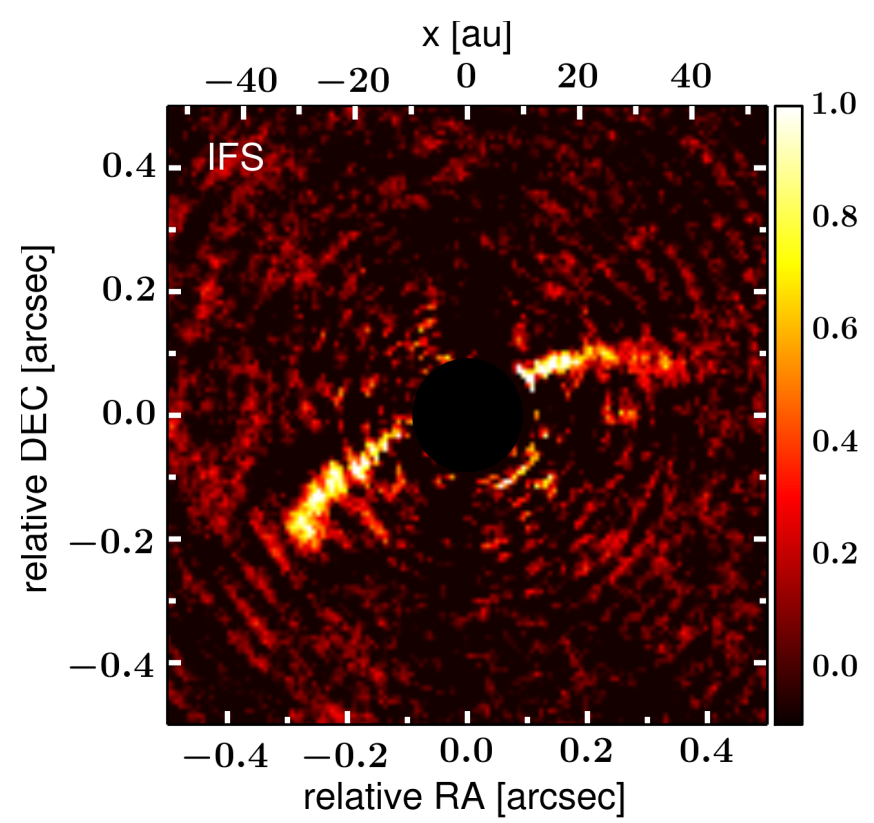

Fig. 4. IFS image after PCA+SDI reduction with 100 modes: median across the entire wavelength range $Y J$. North is up, east is toward the left; the image is normalized to the highest disk brightness and the color scale considers the same dynamical range as in Fig. 3. The inner 0 '.18 region masked by the coronagraph is represented by the black circular area.

\section{Analysis}

\subsection{Radiative transfer modeling of the disk}

We build a radiative transfer model for $\mathrm{T}$ Cha aiming to reproduce the basic structure of its disk. We take earlier efforts (Olofsson et al. 2011, 2013; Huélamo et al. 2015) as a starting point for independent three-dimensional (3D) radiative transfer calculations using the Monte Carlo code RADMC-3D ${ }^{1}$ (Dullemond et al. 2012). We aim to complement the current understanding of the disk geometry by also taking into account our new SPHERE data. RADMC-3D is used to calculate the thermal structure of the dust disk and ray-traced synthetic scattered light images in the NIR. The polarization of RADMC-3D was investigated by Kataoka et al. (2015), who performed a benchmark test against the numerical models presented in Pinte et al. (2009).

\subsubsection{Modeling approach}

The disk around $\mathrm{T}$ Cha is parametrized using constraints obtained from previous analyses of data sets (cf. Olofsson et al. 2011, 2013; Huélamo et al. 2015) and from the new SPHERE observations presented in this paper. We assume the disk to be composed of two spatially separated zones with an inner $\left(r_{\text {in }}\right)$ and outer radius $\left(r_{\text {out }}\right)$ each, a narrow inner disk close to the central star that is responsible for the NIR excess and a more extended outer disk. Inner and outer disks are assumed to be coplanar, since there is no significant evidence for a misaligned inner disk, which would cast shadows onto the outer disk (cf. the cases of HD 142527, Marino et al. 2015 and HD 100453, Benisty et al. 2017). The surface density structure is defined by a power-law

\footnotetext{
1 The RADMC-3D source code and more details are available online at http://www.ita.uni-heidelberg.de/ dullemond/ software/radmc-3d/
}

Table 2. Overview of the best RADMC-3D model parameters.

\begin{tabular}{lcc}
\hline \hline Parameter & Inner disk & Outer disk \\
\hline$r_{\text {in }}[\mathrm{au}]^{*}$ & $0.07^{a}$ & 30 \\
$r_{\text {out }}[\mathrm{au}]^{*}$ & $0.11^{a}$ & 60 \\
$r_{\mathrm{c}}[\mathrm{au}]$ & - & $50^{b}$ \\
$M_{\text {dust }}\left[M_{\odot}\right]$ & $2 \times 10^{-11 a}$ & $9 \times 10^{-5 b}$ \\
$\delta$ & 1 & 1 \\
$\mathrm{H}_{0} / r_{0}$ & $0.02 / 0.1^{a}$ & $4 / 50^{b}$ \\
$\beta$ & 1 & 1 \\
\hline$\left\{a_{\min }, a_{\max }\right\}[\mu \mathrm{m}]^{*}$ & $\{0.01,1000\}$ & $\{0.01,1000\}$ \\
& $\sim 10$ & $\sim 10$ \\
$p$ & -3.5 & -3.5 \\
\hline incl $[\mathrm{deg}]^{*}$ & 69 & 69 \\
PA $[\mathrm{deg}]^{*}$ & 114 & 114 \\
\hline
\end{tabular}

Notes. $\delta$ denotes the exponent of the surface density power-law and $\beta$ corresponds to the disk flaring index. For the radiation source we take the following star parameters: $T_{\text {eff }}=5400 \mathrm{~K}, M=1.5 M_{\odot}, R=1.3 R_{\odot}$, where the star is assumed to be spherical. All parameters marked with an asterisk symbol $\left({ }^{*}\right)$ were varied during the radiative transfer modeling. The grain size distributions as well as inclination and PA were taken to be the same for the inner and outer disk.

References. ${ }^{(a)}$ Olofsson et al. (2013); ${ }^{(b)}$ Huélamo et al. (2015).

profile and an exponential taper at the outer edge of the outer component (e.g., Hughes et al. 2008),

$\Sigma(r)=\Sigma_{0}\left(\frac{r}{r_{\mathrm{c}}}\right)^{-\delta} \exp \left[-\left(\frac{r}{r_{\mathrm{c}}}\right)^{2-\delta}\right]$,

where $r_{\mathrm{c}}$ corresponds to a characteristic radius and $\delta$ denotes the surface density index. For the sake of simplicity, we assume a uniform distribution along the azimuth in our model and concentrate on the radial disk structure. The disk scale height is parameterized radially as $H(r)=H_{0}\left(r / r_{0}\right)^{\beta}$, where $H_{0}$ is the scale height at a reference radius $r_{0}$ and $\beta$ is the flaring index. The vertical density distribution follows a Gaussian profile, so that the dust volume density is given by

$\rho(R, \varphi, z)=\frac{\Sigma(R)}{\sqrt{2 \pi} H(R)} \exp \left(-\frac{z^{2}}{2 H^{2}(R)}\right)$,

where the spherical coordinates $R$ and $z$ can be converted into cylindrical coordinates with $R=r \sin (\theta)$ and $z=r \cos (\theta)$, where $\theta$ is the polar angle. We consider a power-law grain size distribution with an index $p=-3.5, \operatorname{dn}(\mathrm{a}) \propto \mathrm{a}^{p}$ da between a minimum $\left(a_{\min }\right)$ and maximum grain size $\left(a_{\max }\right)$. During our modeling process we use different values for the parameters $a_{\min }$ and $a_{\max }$, where two distributions are eventually find to give an equally good match for the total intensity image (cf. Sect. 4.1.2). The dust is assumed to be a mixture made of silicates (Draine 2003), carbon (Zubko et al. 1996), and water ice (Warren \& Brandt 2008 ) with fractional abundances of $7 \%, 21 \%$, and $42 \%$, consistent with Ricci et al. (2010). The remaining $30 \%$ is vacuum. The opacity of the mixture is determined by means of the Bruggeman mixing formula. The absorption and scattering opacities, $\kappa_{\mathrm{abs}}$ and $\kappa_{\text {scat }}$, as well as the scattering matrix elements $Z_{i j}$ are calculated for spherical, compact dust grains with Mie theory considering the BHMIE code of Bohren \& Huffman (1983). 
The radiative transfer calculations start with computing the dust temperature consistently by means of a thermal Monte Carlo simulation using $10^{7}$ photon packages $^{2}$. Hence, an equilibrium dust temperature is calculated considering the star as the source of luminosity. The main inputs for the radiative transfer modeling are the dust density structure from Eq. (5) and the dust opacities. Full non-isotropic scattering calculations are performed that take multiple scattering and polarization into account. To compare with the observations, synthetic Stokes $I$ intensity images, and Stokes $Q$ and $U$ polarized intensity images are produced at $H$-band $(1.6 \mu \mathrm{m})$ using $10^{8}$ photon packages. These theoretical images are then convolved with a Gaussian PSF with a FWHM of $0{ }^{\prime \prime} 04$ assuming the object to be at $107 \mathrm{pc}$. Moreover, the synthetic total intensity images are run through the MPIA-PCA and SpeCal-PCA processing described in Sect. 2.3 to have a proper comparison. The polarimetric Stokes $Q$ and $U$ images are eventually converted into their azimuthal counterparts $Q_{\phi}$ and $U_{\phi}$. All synthetic images are normalized to the highest disk surface brightness and displayed using the same dynamical range as for the observational data. The coronagraph used in our IRDIS observations is mimicked by masking the inner 0 '.18 of the disk (19.3 au at $107 \mathrm{pc}$ distance).

\subsubsection{Best model}

The inner disk geometry parameters are adopted from Olofsson et al. (2013) and are kept fixed in the modeling process. By adjusting the parameters from Huélamo et al. (2015) we generate the outer disk and run a grid of models exploring a pre-defined parameter space for $r_{\text {in }}, r_{\text {out }}, a_{\min }, a_{\max }$, incl and $\mathrm{PA}$. The fiducial model is defined by the set of parameters that causes a minimization in the residuals between observations and model within the paramater ranges set. For this determination the images of both, data and models, are normalized to the highest flux value outside of the coronagraph. There is, however, no automatic fitting routine since computing tens of thousands of 3D models for the T Cha system is computationally far too expensive. The best parameters are summarized in Table 2. The modeling approach taking into account the new high-contrast SPHERE images allow us to better constrain the position of the inner rim of the outer disk, which we find to be at a significantly larger radius of $\sim 30$ au $(\sim 0 \prime \prime 28)$ compared to earlier work. Hence, the cavity size between the inner and outer disk is correspondingly larger than previously thought (Olofsson et al. 2013). Furthermore, the polarimetric measurements provide us better estimates of the grain sizes.

\section{Synthetic total intensity images}

Figure 5, left panel, shows the synthetic Stokes $I$ image at $\mathrm{H}$-band from the first of our two radiative transfer models. It is produced at a disk position angle of $\mathrm{PA}=114^{\circ}$ and an inclination angle of $i=69^{\circ}$, which is similar to those values derived in Huélamo et al. (2015). Our disk model gives a qualitatively good match with the IRDIS total intensity images from Fig. 3. The bright arc as the dominant source of scattered light is well reproduced and corresponds to forward scattered light from the near side of the inclined disk. The ADI images may, however, be significantly altered by the software processing, which was already shown by Garufi et al. (2016) for the case of HD 100546. This ADI bias is especially important for T Cha,

\footnotetext{
2 Each single package actually represents many photons at once assuming that these photons follow the same path.
}

since the self-subtraction is strong due to the small field rotation and high inclination. Thus, we apply the ADI processing routines to the model image. To do so we process the model image rotated by $70 \mathrm{deg}$ with the raw data considering the same PCA parameters. The middle and right panels of Fig. 5 show the resulting post-processed images depending on the PCA reduction method. The ADI procedure damps the signal of the backside of the disk and introduces a brightness asymmetry along the disk surface. Thus, an original azimuthally symmetric feature can be seen as an asymmetric double-wing structure for a specific disk geometry and orientation. We note here that we additionally favor a physical reason for this asymmetry, since this is also seen in the polarimetric images (cf. Sect. 5.3). The ADI processed model image supports that the geometrical parameters used in our model, in particular the gap size, reproduce the observations nicely.

The fraction of star light scattered off the disk surface layer towards the observer depends on the disk properties (e.g., mass and scale height), but also on dust grain properties that determine the phase function. Dust grains, which are large compared to the wavelength, have strongly forward peaking scattering phase function, while small grains scatter photons almost isotropically. When keeping the minimum dust grain size fixed at $0.01 \mu \mathrm{m}$, a maximum grain size of at least $100 \mu \mathrm{m}$ is requested to match the observations. This serves to reduce the influence of the small grains that are in the Rayleigh limit and absorb radiation much more efficiently than they scatter it. Except for very turbulent disks, one would, however, expect very large grains $(>10 \mu \mathrm{m})$ to settle below the scattering surface. The need for large grains in the disk surface can be avoided by removing the smallest grains. Hence, the minimum and maximum values for the dust grain size distribution in our model are somehow degenerate. An equally good image, that also achieves the desired brightness contrast of the arc with respect to the disk backside, is obtained by using a narrow distribution around $10 \mu \mathrm{m}$. Grains of about ten microns in size are strong forward scatterers in the $H$-band. If even larger particles were primarily present, the forward scattering efficiency would be too strong, and the brightness of the disk's far sides would be too faint. The corresponding synthetic intensity images for the second model and their appearance after the ADI post processing with PCA can be found in Fig. 6 .

\section{Synthetic polarimetric images}

Our results so far demonstrate that we find a quite good model to match the disk geometry of $\mathrm{T}$ Cha. The goal is, however, to also analyze the grain properties compatible with the polarimetric data. For scattering in the Rayleigh and Mie regime, that is, for grains with sizes smaller than or approximately equal the wavelength $(2 \pi a \lesssim \lambda)$, maximum polarization is expected along a scattering angle of $90 \mathrm{deg}$. The phase function $Z_{11}$, the scattering matrix element $-Z_{12}$ and the degree of polarization $-Z_{12} / Z_{11}$ of the dust grains used in our radiative transfer models are shown as a function of the scattering angle $\theta$ in Fig. 7. Comparing those quantities for both models allows us to rule out the first model covering a wide range of grain sizes from 0.01 to $1000 \mu \mathrm{m}$. This, rather, produces maxima in polarized intensity along the semimajor axis (see Appendix A), which is clearly not observed in the SPHERE PDI data from Fig. 1. Although one can recognize an extreme forward peak in $-Z_{12}$, the resulting peak in polarized intensity is hidden behind the coronagraph. For our second model with grains of $\sim 10 \mu \mathrm{m}$ the phase function is also dominated by small-angle scattering as seen in the $Z_{11}$ plot, but the $-Z_{12}$ curve has a strong peak at small angles of $\sim 10^{\circ}$. This leads 

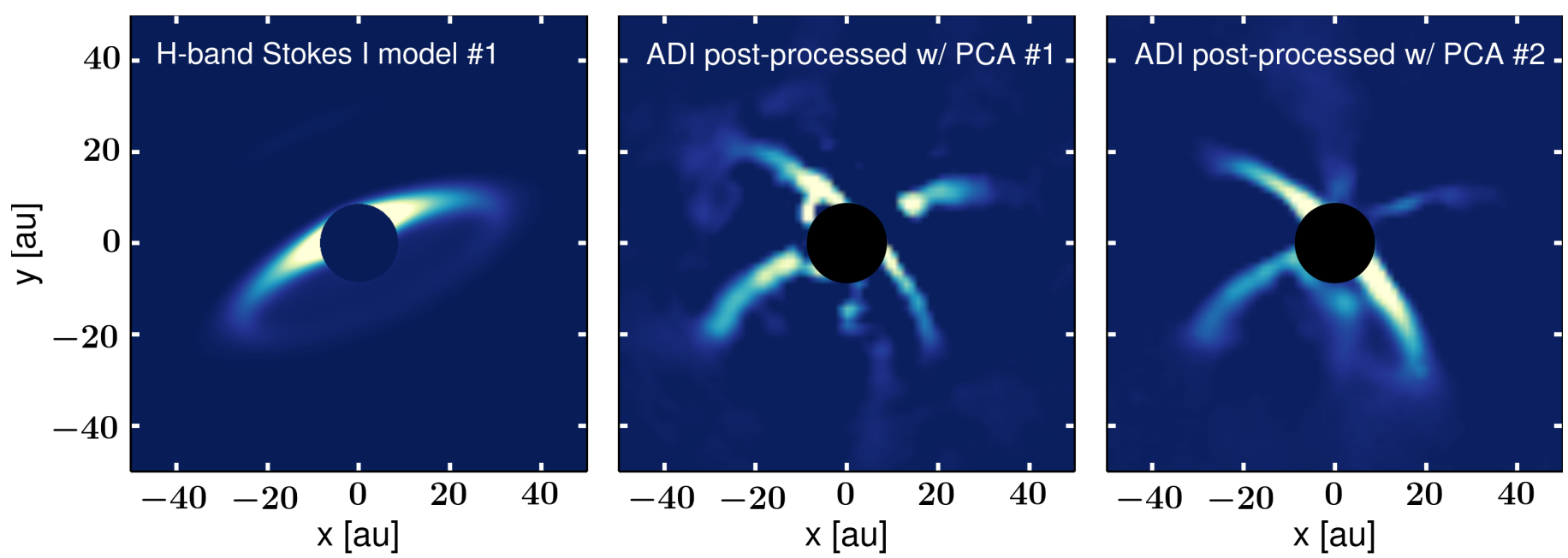

Fig. 5. Synthetic total intensity images from our radiative transfer model \#1 considering a MRN-like power-law grain size distribution with $a_{\min }=0.01 \mu \mathrm{m}$ and $a_{\max }=1000 \mu \mathrm{m}$. The left panel shows the theoretical Stokes $I$ image convolved with a Gaussian PSF with FWHM of 0'”04 (at $107 \mathrm{pc}$ distance). The middle and right panels show the theoretical model image at $70^{\circ}$ processed together with the raw DBI data by the different PCA methods as described in Sect. 2.3. The central 0'.18 of the image are masked to mimic the effect of the coronagraph on the observations. The units are arbitrary, but the dynamical range of the color bar is taken the same as in Fig. 3.
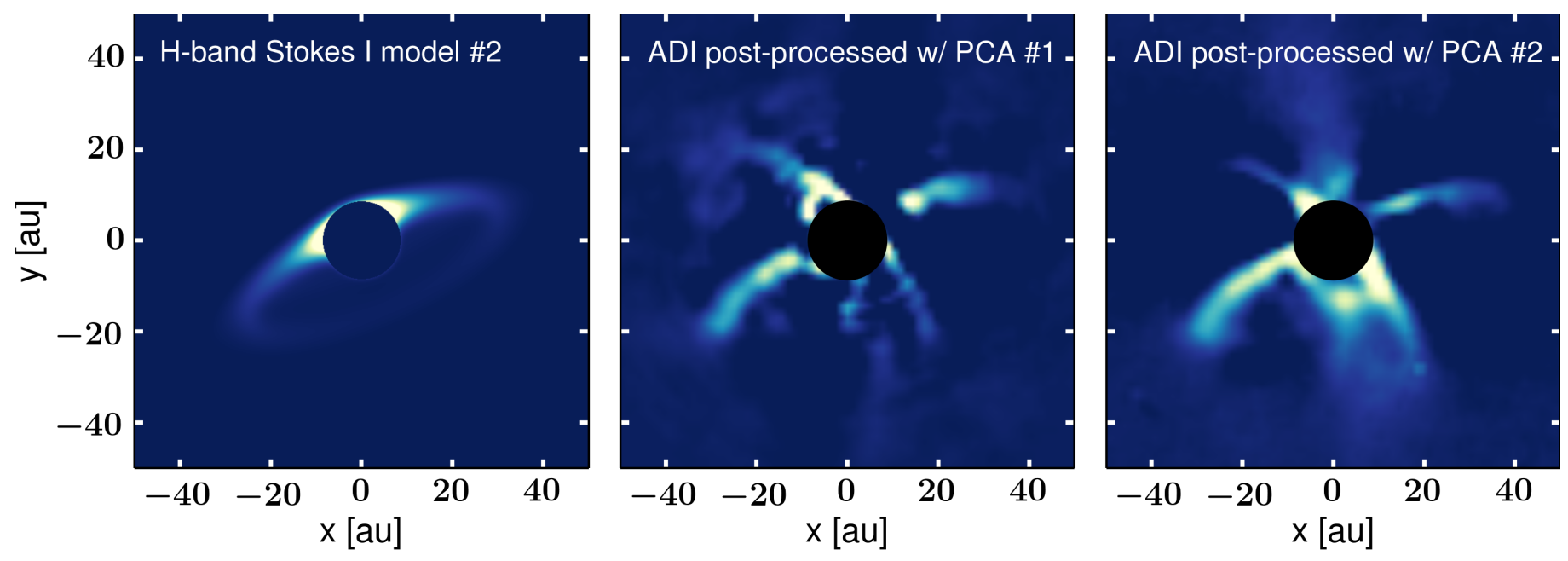

Fig. 6. Synthetic total intensity images from our radiative transfer model \#2 considering a grain size distribution narrowly peaked around $\sim 10 \mu \mathrm{m}$. The layout and color scale is identical to Fig. 5.
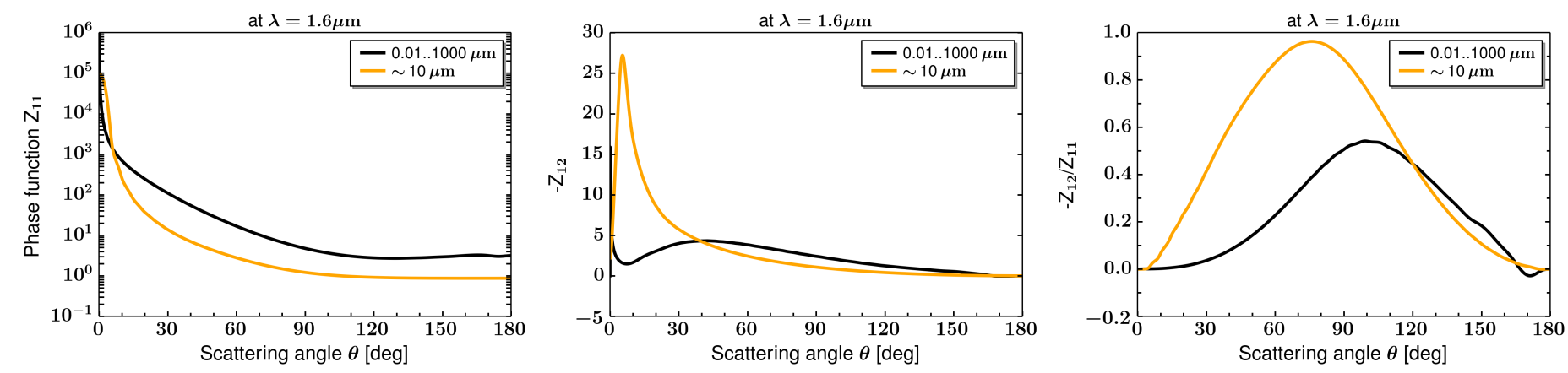

Fig. 7. Phase functions $Z_{11},-Z_{12}$ and degree of polarization $-Z_{12} / Z_{11}$ of the dust grains dependent on the scattering angle $\theta$ and calculated at $\lambda=1.6 \mu \mathrm{m}$. Model \#1 is represented by the black line, model \#2 by the orange curve.

to the spatial shift of brightness maxima away from the semimajor axis (i.e., scattering at $90 \mathrm{deg}$ ), meaning that the maximum polarized intensity occurs at the forward scattering position. This is in good agreement with our polarimetric SPHERE observations.
Figure 8 shows the synthetic $Q_{\phi}$ and $U_{\phi}$ images at $H$-band for the second model, with a disk position angle of $\mathrm{PA}=114^{\circ}$ and an inclination of $i=69^{\circ}$; both determined from the fit to the total intensity image. The $Q_{\phi}$ image is dominated by large positive signal, which is consistent with forward scattering from the 

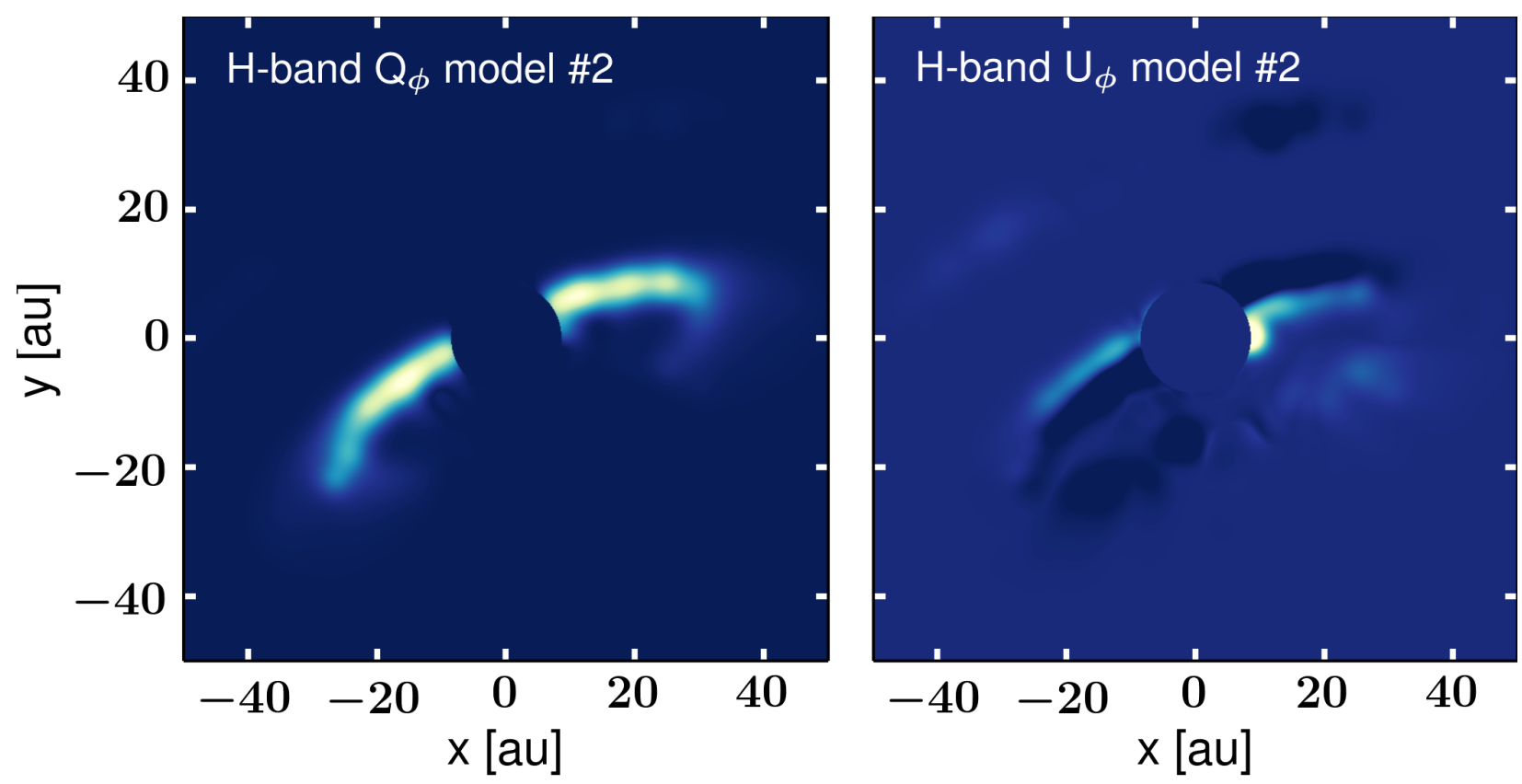

Fig. 8. Synthetic $Q_{\phi}$ (left) and $U_{\phi}$ (right) images at $H$-band. They are convolved with a Gaussian PSF with FWHM of 0 '04 (at 107 pc distance). The color scale is arbitrary, the dynamical range is similar as in Fig. 1. Negative values of $U_{\phi}$ are saturated at dark blue color.
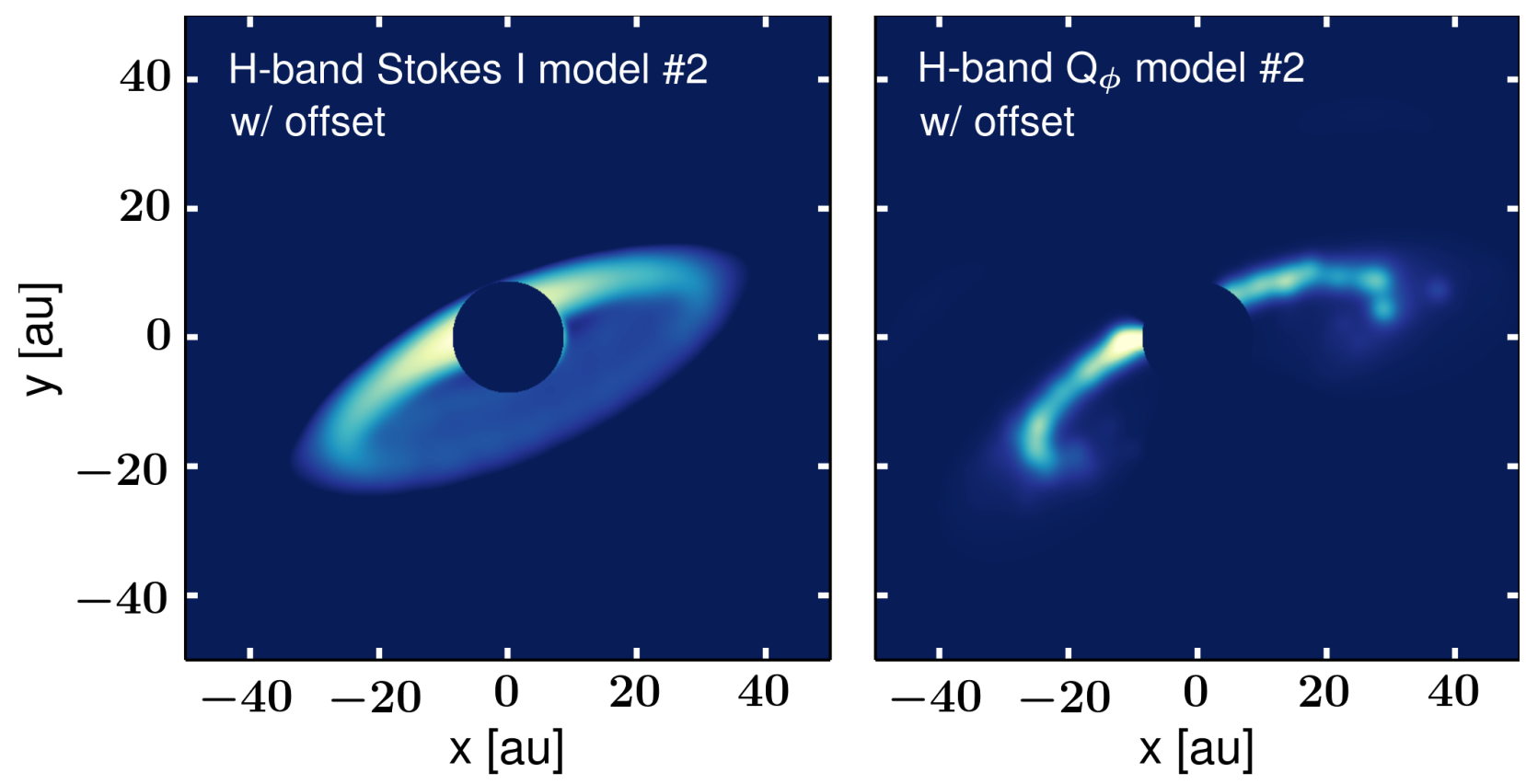

Fig. 9. Synthetic Stokes $I$ (left) and $Q_{\phi}$ (right) images at $H$-band of model \#2, where the star is slightly offset from its original central position along the semi-major axis. The images are convolved with a Gaussian PSF with FWHM of 0’’04 (at $107 \mathrm{pc}$ distance). The color scales are identical to Figs. 5 and 8, respectively.

close edge of the disk. The small-scale brightness blobs could be due to self-scattering of thermal emission or the result of multiple scattering treatment in the radiative transfer calculations. Monte Carlo noise can be ruled out as the source of these features since the best models were also run with a higher number of photon packages $\left(10^{9}\right)$ for testing, confirming that our calculations are converged. Similar to the observed $U_{\phi}$ image, the $U_{\phi}$ model image shows an alternation of positive (white) and negative (dark blue) signal, although the exact geometry appears different. The extension of the south-east lobe with negative signal is comparable to that in the observational image in Figs. 1 (bottom left panel) and 2. The positive signal is a bit less pronounced in our calculated model. Since the $U_{\phi}$ signal in the observational image can be substantially influenced by noise, instrumental effects, and the data reduction procedure, which is not included in our modeling, such a deviation was to be expected. The $U_{\phi} / Q_{\phi}$ peak-to-peak value for the best model is about $15 \%$, which is still in very good agreement with the observations (9\% and 14\%), but lower than calculated in the study by Canovas et al. (2015) on non-azimuthal linear polarization. For their models and in our RADMC-3D calculations we consider a full treatment of polarized scattering off randomly oriented particles. Due to the absence of any instrumental influence on the polarization, the $U_{\phi}$ signal visible in the model images should be primarily connected 
to multiple scattering events happening in the disk. However, the contribution of multiple scattering strongly depends on the disk inclination, the grain population and the mass of the disk. In Canovas et al. (2015) the signal in $U_{\phi}$ reaches up to $50 \%$ of the $Q_{\phi}$, but only for an inclination of $70 \mathrm{deg}$, a grain size distribution with $a_{\min , \max }=(5,1000) \mu \mathrm{m}$, and a disk significantly more massive than assumed for our T Cha model. A higher disk mass produces more scattering events as simply more scattering particles are available. Furthermore, the higher scattering efficiency of the grains relative to their absorption efficiency results in stronger multiple scattering signature in the models of Canovas et al. (2015). These effects can explain the discrepancy to our $U_{\phi} / Q_{\phi}$ peak-to-peak value of only $15 \%$.

\section{East-west brightness asymmetry}

The clear asymmetry in brightness along the inner edge of the outer disk from the observations is naturally not produced with our symmetric disk model with spatially invariant dust properties. A slightly offset disk is one possibility for explaining the origin of the asymmetry and we explore this scenario in the following. We take our best axisymmetric model and slightly displace the star along the semi-major axis with respect to its original central position, while keeping the general disk structure unchanged. This is directly implemented into the radiative transfer code and not performed in a post-processing manner. A grid of additional models is computed, where the magnitude of the physical offset between the center of the T Cha disk and the position of its host star is changed between 0.5 and $2.5 \mathrm{au}$. We are only interested whether such a scenario is principally reliable, so we abstain from a fitting procedure. A value of $x=2.1 \mathrm{au}$, where $x$ is measured along the disk's semi-major axis, represents a reasonable match. The offset we apply is equivalent to a disk eccentricity of $e \approx 0.07$. This way a brightness contrast between the east and west sides of 2 (Stokes $I$ ) and 3 (Stokes $Q_{\phi}$ ) can be reached (see Fig. 9), consistent with the observational constraints. Other possible scenarios for the brightness asymmetry are discussed in Sect. 5.3.

\subsection{Point source analysis}

One candidate companion (CC) is detected in the IRDIS field of view (Fig. 10), whereas no point-like sources are found in the IFS image. The speckle pattern is reduced in each frame of the sequence by subtracting an optimized reference image calculated by the TLOCI algorithm (Marois et al. 2010) implemented in SpeCal. We estimate the astrometry and photometry of this companion candidate using the calibrated unsaturated PSF (Galicher \& Marois 2011) to remove biases. First, we roughly estimate the flux and position of the source in the TLOCI image. The SpeCal pipeline then creates a data cube of frames that only contain the unsaturated PSF at the candidate position on the detector, accounting for the field-of-view rotation in each frame. The TLOCI coefficients used to generate the TLOCI image where the candidate is detected are applied on the candidate data cube. The resulting frames are rotated to align north up. The median of these frames provides the estimation of the candidate image in the TLOCI image. We then adjust the estimated image subpixel position and its flux to minimize the integrated flux of the difference between the real and estimated candidate images. We use a $3 \times F W H M$ diameter disk for the minimization. The $1 \sigma$ error bars are the required excursions in position or in flux to increase the minimum residual flux by a factor of

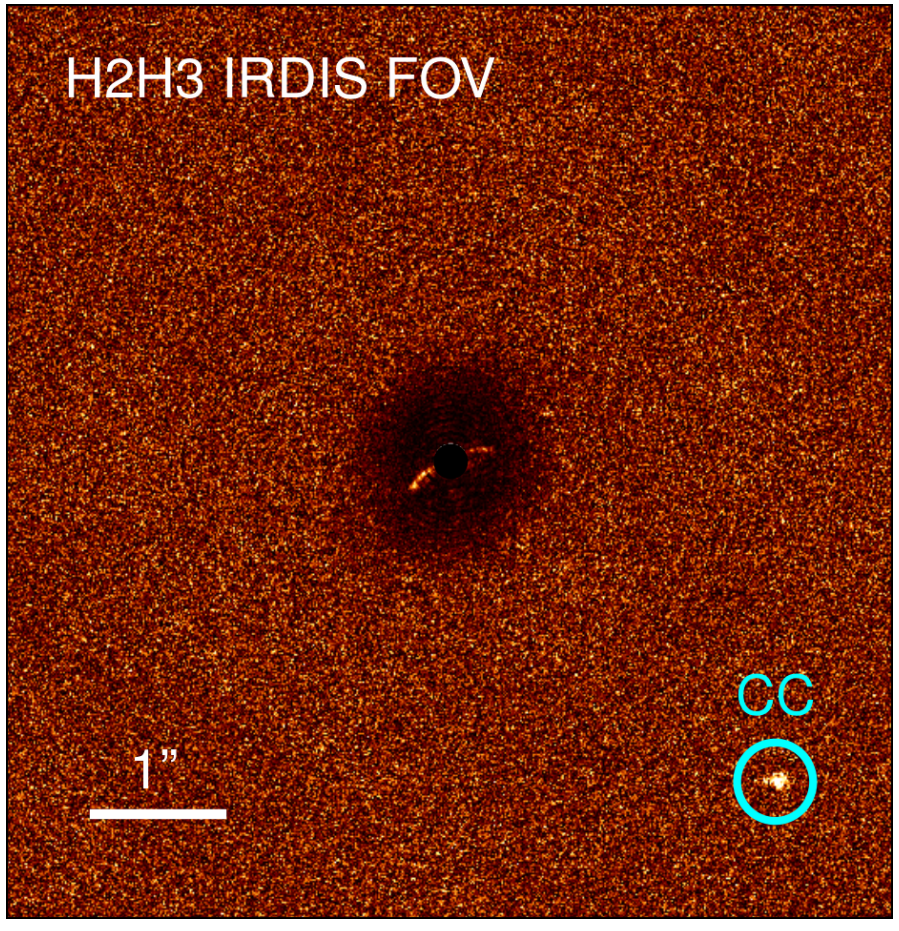

Fig. 10. Signal-to-noise ratio map of the PCA reduction (\#2) of the IRDIS $\mathrm{H} 2 \mathrm{H} 3$ data. The point source considered as a companion candidate (CC) is marked with a circle.

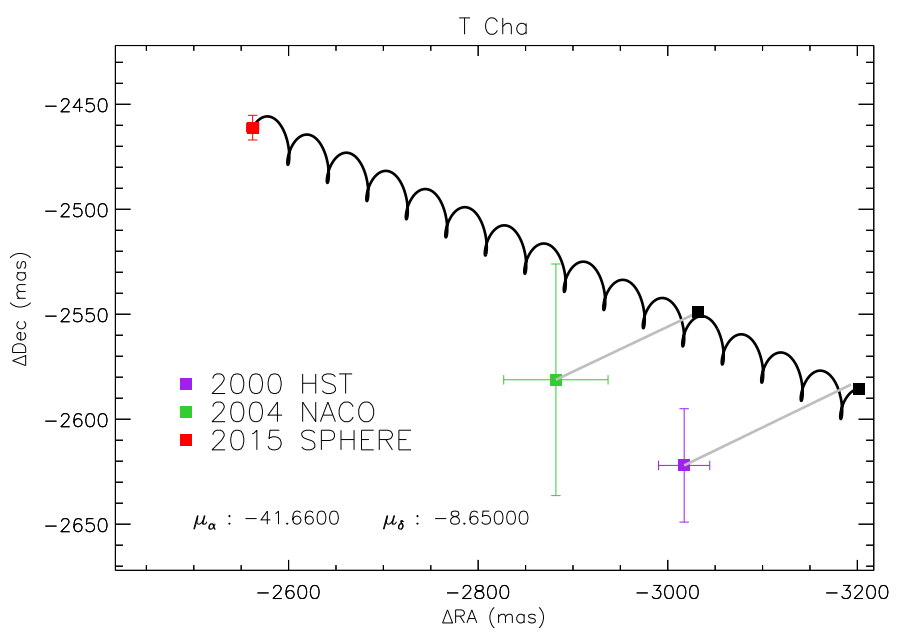

Fig. 11. Relative astrometry of the companion candidate labeled as "CC" in Fig. 10 measured in SPHERE, NACO and HST data. The black solid line displays the motion of the companion if co-moving and the black squares are the positions expected at the time of HST and NACO observations.

$\sqrt{1.15}$ (cf. Galicher et al. 2016, Galicher et al., in prep.). We empirically determine this factor running tests on sequences, in which we inject known fake planets. Using the calibrated unsaturated PSF, we also estimate the TLOCI throughput in all TLOCI sections following a procedure similar to the one used for the candidate position and flux estimation. The images were thus flux calibrated. The systematic errors for the astrometry of the detected companion candidate include the uncertainties on the pixel scale, North angle, frame centering using the satellite spots, accuracy of the IRDIS dithering procedure, anamorphic correction and SPHERE pupil offset angle in pupil-tracking mode (Vigan et al. 2016; Maire et al. 2016). The calibration uses pixel 


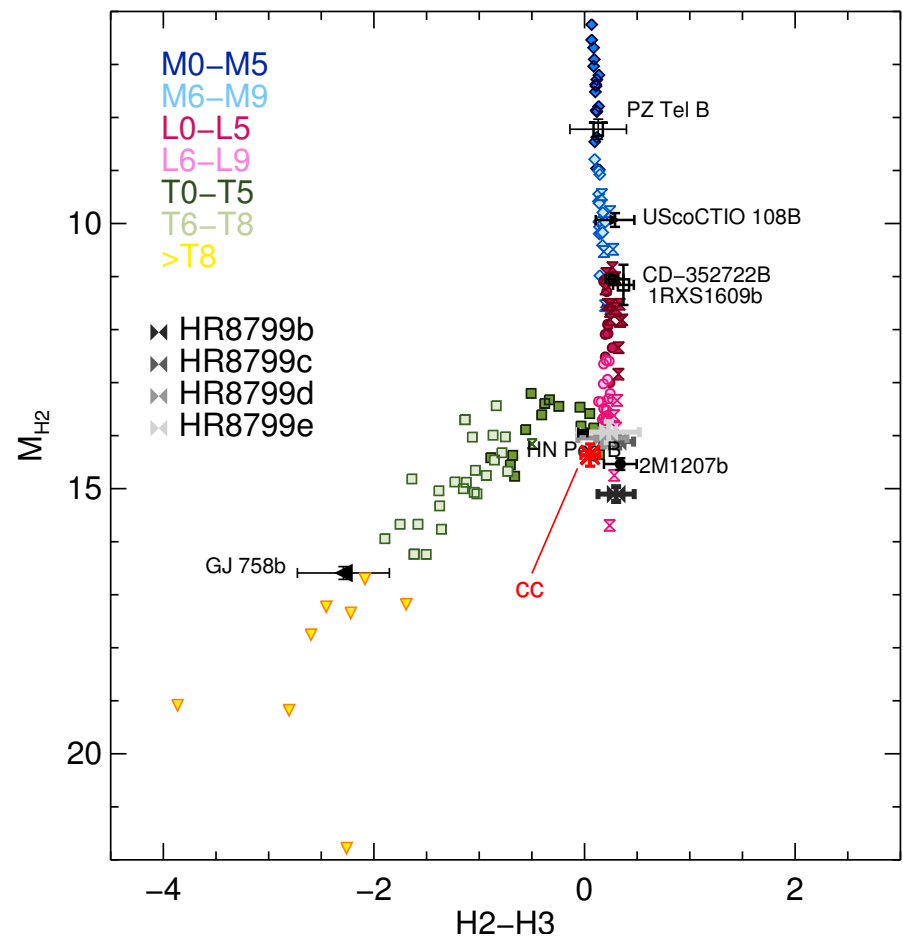

Fig. 12. Color-magnitude diagram displaying our candidate companion, which is marked in red and labeled with CC, compared to known substellar field (colored symbols) and young objects. Note that this plot assumes that CC is at the same distance as the star. Since CC is eventually classified as a background object based on a common proper motion test (cf. Fig. 11), it is likely located much further.

Table 3. Astrometry and photometry relative to the star of the companion candidate in the T Cha system.

\begin{tabular}{lcc}
\hline \hline \multicolumn{3}{c}{ IRDIS companion candidate } \\
\hline Filter & $H 2$ & $H 3$ \\
$\lambda[\mu \mathrm{m}]$ & 1.593 & 1.667 \\
Contrast [mag] & $11.65 \pm 0.04$ & $11.60 \pm 0.04$ \\
SNR & 31.1 & 31.4 \\
RA [mas] & $-2560.3 \pm 6.1$ & $-2562.8 \pm 6.0$ \\
DEC [mas] & $-2460.1 \pm 5.7$ & $-2461.29 \pm 5.7$ \\
Separation [mas] & $3551.9 \pm 8.2$ & $3552.1 \pm 8.1$ \\
PA [deg] & $226.14 \pm 0.18$ & $226.16 \pm 0.18$ \\
\hline
\end{tabular}

Table 4. Relative astrometry of the companion candidate for different instrumental data.

\begin{tabular}{lcc}
\hline \hline & NACO & SPHERE \\
\hline Date & 5 March 2004 & 30 May 2015 \\
JD & 2453070 & 2457173 \\
Separation [mas] & $3868.9 \pm 55.0$ & $3552.6 \pm 10.7$ \\
PA [deg] & $228.2 \pm 0.8$ & $226.15 \pm 0.18$ \\
\hline
\end{tabular}

Notes. The NACO data were published in Chauvin et al. (2010).

scales of $(12.255 \pm 0.009)$ mas/pix and $(12.251 \pm 0.009) \mathrm{mas} / \mathrm{pix}$ for the $H 2$ and $H 3$ filters, respectively, and a true North offset of $(-1.712 \pm 0.063)^{\circ}$ is considered (Maire et al. 2016).

$\mathrm{CC}$ is located at a separation of $(3.55 \pm 0.01)^{\prime \prime}$ with contrast $\left(\Delta m_{\mathrm{H}}=11.63 \pm 0.04\right) \mathrm{mag}$ (see Table 3$)$. This same companion was already detected by Chauvin et al. (2010) with $m_{K}=(11.4 \pm 0.1) \mathrm{mag}$ and is also present in HST data taken in coronagraphic mode with STIS in March 2000. Combining the new position measured from SPHERE with the old data we rule out this object as being gravitationally bound to $\mathrm{T}$ Cha, because the motion observed over these 15 years is too large to be explained by a Keplerian orbit around this star; it is therefore a contaminant object. Given that the T Cha proper motion is $\mu_{\alpha}=(-41.66 \pm 0.2) \mathrm{mas} / \mathrm{yr}$ and $\mu_{\delta}=(-8.65 \pm 0.19) \mathrm{mas} / \mathrm{yr}$ (Gaia Collaboration 2016), we also notice that CC has a high relative proper motion with respect to a background object (Fig. 11). For completeness, we show the CMD in Fig. 12. We note that this plot assumes that $\mathrm{CC}$ is at the same distance as $\mathrm{T}$ Cha, since its actual distance is unknown. This is rather unlikely based on our previous conclusion that it is not physically associated with $\mathrm{T}$ Cha. $\mathrm{CC}$ is likely located much further, and thus, likely intrinsically much brighter than an object at the L-T transition. Given the $H 2-H 3$ color $\sim 0$, we conclude that this object could be either a floating brown dwarf or a low mass star of the galactic thick disk or halo.

\subsection{Detection limits on substellar companion candidates}

The IRDIS detection limits for point sources are determined using the TLOCI data reduction. We estimate the $5 \sigma$ noise level, where $\sigma$ is the azimuthal robust deviation of the residual flux in annuli of $\lambda / \mathrm{D}$ width rejecting pixels with no flux. Finally, the $5 \sigma$ noise levels are divided by the stellar flux estimated from the unsaturated images. The maximum contrast reached with IFS is obtained by applying the PCA technique. The contrast limits are estimated by an azimuthal standard deviation, that is, between pixels at the same separation from the star, for each angular separation, corrected by the star flux (obtained from the off-axis PSF images taken immediately before and after the coronagraphic observations) and the algorithm throughput (using synthetic companions injected into the data before the data processing as described above).

In Fig. 13 the contrast curves obtained for the different data sets are shown. The IRDIS data give a $5 \sigma$ contrast for a separation larger than $1.0^{\prime \prime}$ of greater than $12.5 \mathrm{mag}$ and $12.6 \mathrm{mag}$ in the $H 2$ and $H 3$ bands, respectively. Compared with NACO $K_{\mathrm{s}}$ band results (cf. Chauvin et al. 2010), these observations are deeper by more than three magnitudes at a separation of 0.7 , that is, at the outer edge of the NACO coronagraph, while the contrast values at wider separations are comparable. IFS is deeper in contrast for separations closer than $00^{\prime \prime} 6$ and gives $12 \mathrm{mag}$ in the $Y J$ band at a separation of $\sim 0^{\prime \prime} 7$, assuming a gray contrast between the two objects.

Using the theoretical atmospheric models AMES-COND (Allard et al. 2003) we convert the contrast limits into upper limits on the mass of possible objects orbiting around $\mathrm{T}$ Cha. These models are valid for $T_{\text {eff }}<1400 \mathrm{~K}$ and consider that the dust immediately rains out from the photosphere after its formation. We assume a system age of $7 \mathrm{Myr}$ (Torres et al. 2008). This leads to a mass limit of $\sim 8.5 M_{\text {jup }}$ in the innermost regions $\left(\sim 00^{\prime \prime} 1-00^{\prime \prime} 2\right)$, decreasing to $\sim 2 M_{\text {jup }}$ for a separation between 0 "'4 and 5'”0. Our new SPHERE observations, therefore, improve the NACO mass limits especially up to $\sim 1.5^{\prime \prime}$. Both, the contrast and mass curve are cut at $0 \prime \prime 12$. The whole coronagraph system (apodizer, mask, stop) produces a radial transmission profile, which has not been accounted for in the derivation of the detection limits. The effect is visible at the region near the edge of the mask plus $\lambda / D$, thus, we exclude the inner $00^{\prime \prime} 12$. Furthermore, we note that the detection limits represent an average value around the star, which 

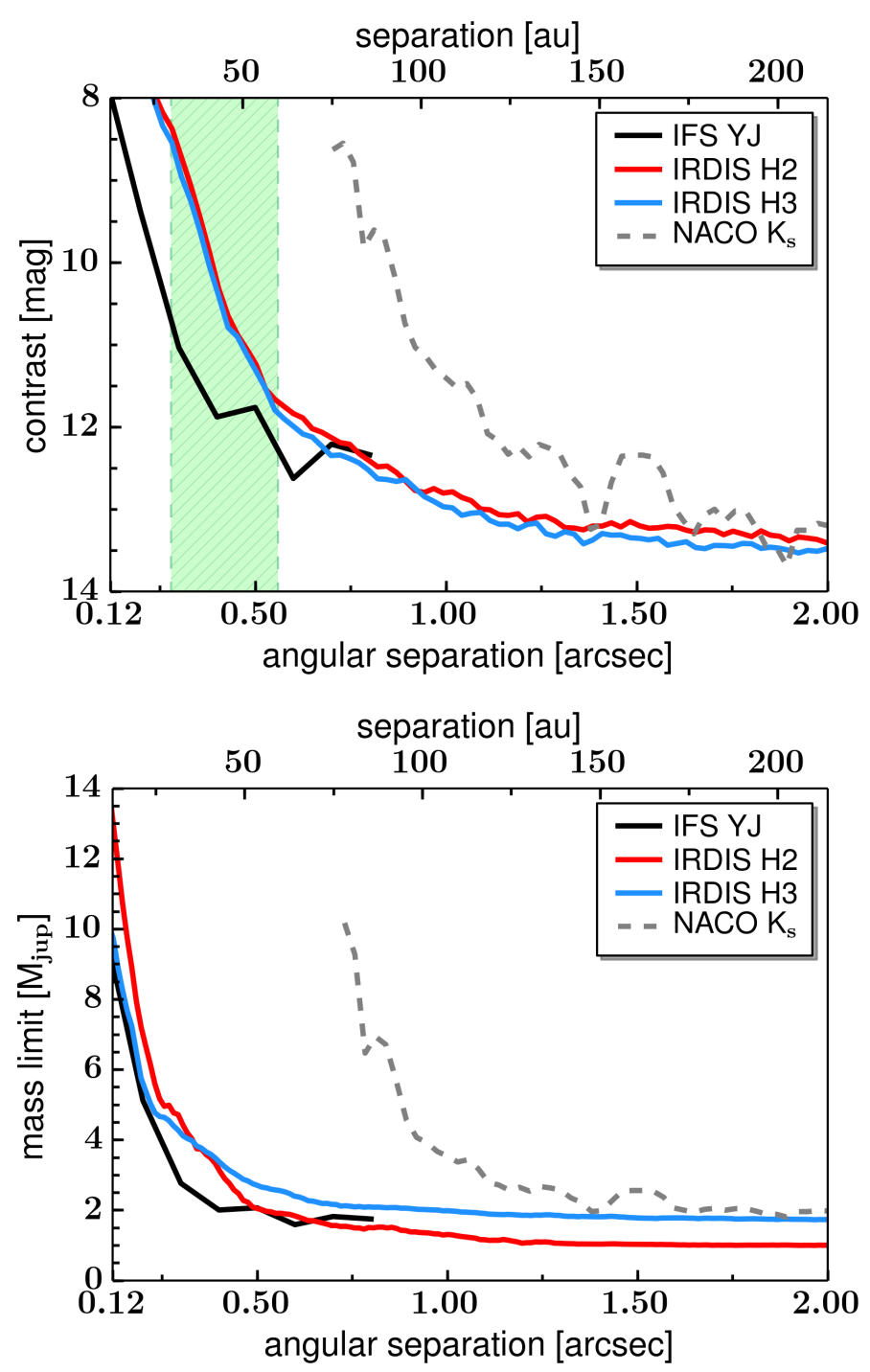

Fig. 13. Contrast curves and companion mass limits derived for IFS (black) after applying PCA, and for IRDIS $H 2$ and $H 3$ bands (red and blue, respectively) from the TLOCI reduction (\#3). Both curves have a lower cut at $0 \prime 12$. The detection limits from NACO $K_{\mathrm{s}}$ band data are given for comparison (gray dashed line, inner cut at $0{ }^{\prime \prime} \cdot 7$ ). The green striped rectangle denotes the area for which the contrast values might be slightly affected by the presence of the disk in scattered light.

might be affected by the disk signal at the location of the disk. However, we expect this effect to be small given the rather compact nature of the disk around $\mathrm{T}$ Cha.

\section{Discussion}

\subsection{Disk geometry}

Our analysis and modeling of the SPHERE data set confirms that the disk around $\mathrm{T}$ Cha consists of an inner disk part and an outer disk part, separated by a cavity. Compared to the previous study by Olofsson et al. (2013) we find the small dust cavity size to be larger by a factor of $\sim 2$. Besides, it is even larger than the mm dust cavity of 20 au estimated in Huélamo et al. (2015). This is rather unexpected, as the dust trapping scenario for transition disks is supposed to work such that bigger dust is trapped at a ring located outside of the small dust/gas cavity edge (see e.g., Pinilla et al. 2012; van der Marel et al. 2015). This possible contradiction could be, however, due to uncertainties in the model fitting of data with low resolution by Huélamo et al. (2015). An inclination angle of $\sim 69^{\circ}$ and PA of $\sim 114^{\circ}$ best match our SPHERE observations, which is in agreement with Huélamo et al. (2015). Our new optical and near-infrared data do not, however, help us to constrain the outer disk radius. In our radiative transfer model we considered a tapered density profile for the dust density description of the outer disk, meaning that the surface density falls off gradually and hence, there is a smooth decrease of the dust mass per radius bin. However, simultaneously reproducing the gas and dust components of the disk remains challenging, and including this in our modeling effort is beyond the scope of this paper.

\subsection{Grain properties}

To simultaneously match the total intensity and polarimetric images obtained during our SPHERE observations, intermediate sized grains of $\sim 10 \mu \mathrm{m}$ must be present in the disk. This provides a better match with the observed properties of the disk than dust distributions covering several orders of magnitudes in size or a narrow distribution peaking at (sub-)micron size. This is in accordance with current grain growth models producing systematically larger grains, although we cannot guarantee that $\sim 10 \mu \mathrm{m}$ grains are located at the upper surface layer. By means of scattered light observations in the NIR we only trace the disk surface where the micron-sized grains are located for sure. Compact grains of a few tens of microns are expected to start settling down toward the disk midplane. The efficiency and timescale of vertical mixing depends, however, on the level of turbulence in disks which is still uncertain. With strong turbulence (high $\alpha$-viscosity, Shakura \& Sunyaev 1973) all grain sizes are better mixed. Thus, even larger grains can be present in the disk surface where they can contribute to the scattering. Furthermore, the amount of porosity of dust grains is unknown and still debated (e.g., Ossenkopf 1993; Dominik \& Tielens 1997; Kataoka et al. 2014). For fractal aggregates with high porosity the phase function is supposed to differ (Tazaki et al. 2016), which might alter our grain picture for $\mathrm{T}$ Cha. A larger porosity for the same grain size might reduce the settling, where the size of the monomers still determines the absorption and scattering opacities. As shown by Min et al. (2012), the appearance of a disk in scattered light could be different depending on the fraction of fluffy aggregated dust particles compared to compact grains contained in the disk. We also note that very large grains ( $\mathrm{mm}$ size) are indeed also expected to be present in the midplane in order to match the (sub-)mm data (cf. Huélamo et al. 2015).

\subsection{Brightness asymmetry along the disk surface}

The intensity and polarized intensity distributions observed for $\mathrm{T}$ Cha are asymmetric with respect to the minor axis of the disk. Similar brightness variation has also been detected in other disks, such as RY Tau (Takami et al. 2013) and AK Sco (Janson et al. 2016). In our radiative transfer modeling we explored the origin of the east-west asymmetry seen along the semi-major axis in the SPHERE observations by looking into the simplest possibility of a slightly offset disk. We approximate such an eccentric disk by calculating scattered light images of an azimuthally symmetric disk, but introducing an offset between the disk center and the star. A planetary companion on an eccentric orbit could shape the outer disk into an eccentric disk, causing the offset. Keeping $\mathrm{T}$ Cha's stellar properties as the photon source in the radiative transfer code, but adding a positional offset, already reproduces well the observed asymmetry. 
However, alternative explanations for the east-west brightness difference cannot be ruled out, and several effects may interact. Another idea is that an asymmetry in the inner disk or at the gap edges can lead to illumination effects helping to explain the dips in scattered light. The circumstellar disk around $\mathrm{T}$ Cha may be actually still in an early stage of planetary formation. Thus, a dense dust clump formed in the inner, densest parts of the disk, or an already formed yet undetected planetary perturber below the detection limit, could cause this asymmetry. However, this scenario also raises the question of whether such an anisotropy is indeed stationary or moves with the local Keplerian velocity. A third scenario deals with spatially variant dust properties leading to a different scattering efficiency, which is especially related to grain size, structure, and composition. A possibility would be that unequal dust grain size distributions are present in the east and west wings of the disk, whose origin, however, remains unexplained. A fourth possible scenario leading to shadows in the outer disk is an inner disk significantly tilted with respect to the outer disk's plane. However, we find this scenario unlikely, since this arrangement would rather lead to relatively sharp, dark lanes, which are not apparent in our T Cha images.

\section{Conclusions}

We have carried out VLT/SPHERE optical and NIR observations in polarimetric differential imaging mode with SPHERE/ZIMPOL in $V B B$ and SPHERE/IRDIS in $H$-band of the evolved transition disk around the T Tauri star T Cha. Alongside the polarimetric observations, intensity images from IRDIS $\mathrm{H} 2 \mathrm{H} 3$ dual-band imaging with simultaneous spectro-imaging with IFS in $Y J$-band were obtained. The disk is clearly detected in all data sets presented in this work and resolved in scattered light with high angular resolution, allowing us to review the current understanding of the disk morphology and surface brightness. The basic structure of a classical transition disk previously reported by interferometric and (sub-)mm studies, has been confirmed. We developed a radiative transfer model of the disk including a truncated power-law surface density profile. The conclusions of this paper are summarized below.

1. Our RADMC-3D radiative transfer model with updated disk parameters accounts well for the main geometry of the disk, the cavity, and the outer disk with its bright inner rim located at 0 '!28 $(\sim 30 \mathrm{au})$. This is significantly further out than previously estimated. A disk inclination of $\sim 69^{\circ}$ and a position angle of $\sim 114^{\circ}$ matches the SPHERE data sets best.

2. We confirm that the dominant source of emission is forward scattered light from the near edge of the disk, given the high disk inclination. While small grains in the Rayleigh limit scatter photons rather isotropically and absorb very efficiently, large dust grains with sizes $(2 \pi \mathrm{a}>\lambda)$ have strong forward scattering properties. This demands a certain range of grain sizes to be present in the disk. We found that a power-law distribution with $a_{\min }=0.01 \mu \mathrm{m}$ and $a_{\max }=$ $1000 \mu \mathrm{m}$ reproduces the total intensity observations well, but fails to be consistent with the polarimetric images. Thus, we propose a dominant grain size in the disk of $\sim 10 \mu \mathrm{m}$. Such grains bring the desired amount of forward scattering and lead to a model that is in accordance with the complete SPHERE data set presented. We note that we restricted ourselves to the analysis of Mie theory and spherical compact grains. However, for aspherical aggregates with high porosity the phase function is supposed to differ, which might alter our grain picture for $\mathrm{T}$ Cha.
3. Our highly inclined disk model shows a significant $U_{\phi}$ signal at $H$-band, which is in accordance with the observational $U_{\phi} / Q_{\phi}$ peak-to-peak value of $14 \%$ and theoretical studies on multiple scattering events. The exact geometrical $U_{\phi}$ pattern observed with IRDIS is not reproduced, but the alternating structure of positive and negative lobes is well recognizable.

4. The brightness asymmetry between the east and west sides can be reproduced with a slight offset of the star's position, representing a disk eccentricity of $e \approx 0.07$. A planetary companion on an eccentric orbit could force the outer disk to become eccentric, causing this offset. However, a locally different grain size distribution and therefore a change of the scattering properties, or illumination effects due to asymmetric structures in the inner disk could also contribute to the brightness contrast observed.

5. A previously known companion candidate is detected in the IRDIS field of view at a separation of $(3.54 \pm 0.01)^{\prime \prime}$ with contrast $m_{\mathrm{H} 2}=(11.63 \pm 0.04)$ mag. We, however, rule out the possibility that this object is bound and, thus, conclude that it is not part of the T Cha system.

6. Our analysis rules out the presence of a companion with mass larger than $\sim 8.5 M_{\text {jup }}$ between 0 '! 1 and $00^{\prime \prime} 3$ from the central star, and larger than $\sim 2 M_{\text {jup }}$ for wider separations. There could still be lower-mass planets in the outer disk regions and/or planets in the very inner disk.

Acknowledgements. We would like to thank the ESO Paranal Staff for their support during the observations. We are very grateful to C.P. Dullemond for insightful discussions. A.P. is a member of the International Max Planck Research School for Astronomy and Cosmic Physics at Heidelberg University, IMPRSHD, Germany. INAF-Osservatorio Astronomico di Padova acknowledges support from the "Progetti Premiali" funding scheme of the Italian Ministry of Education, University, and Research. M.L., M.B., F.M. and C.P. acknowledge funding from ANR of France under contract number ANR-16-CE31-0013. J.O. acknowledges support from ALMA/Conicyt Project 31130027, and from the Millennium Nucleus RC130007 (Chilean Ministry of Economy). SPHERE is an instrument designed and built by a consortium consisting of IPAG (Grenoble, France), MPIA (Heidelberg, Germany), LAM (Marseille, France), LESIA (Paris, France), Laboratoire Lagrange (Nice, France), INAF-Osservatorio di Padova (Italy), Observatoire de Genève (Switzerland), ETH Zurich (Switzerland), NOVA (Netherlands), ONERA (France) and ASTRON (Netherlands), in collaboration with ESO. SPHERE was funded by ESO, with additional contributions from CNRS (France), MPIA (Germany), INAF (Italy), FINES (Switzerland) and NOVA (Netherlands). SPHERE also received funding from the European Commission Sixth and Seventh Framework Programmes as part of the Optical Infrared Coordination Network for Astronomy (OPTICON) under grant number RII3-Ct-2004-001566 for FP6 (2004-2008), grant number 226604 for FP7 (2009-2012) and grant number 312430 for FP7 (2013-2016).

\section{References}

Absil, O., Milli, J., Mawet, D., et al. 2013, A\&A, 559, L12

Ageorges, N., Fischer, O., Stecklum, B., Eckart, A., \& Henning, T. 1996, ApJ, 463, L101

Alcala, J. M., Covino, E., Franchini, M., et al. 1993, A\&A, 272, 225

Allard, F., Guillot, T., Ludwig, H.-G., et al. 2003, in Brown Dwarfs, ed. E. Martín, IAU Symp., 211, 325

Amara, A., \& Quanz, S. P. 2012, MNRAS, 427, 948

Andrews, S. M., Wilner, D. J., Espaillat, C., et al. 2011, ApJ, 732, 42

Avenhaus, H., Quanz, S. P., Schmid, H. M., et al. 2014, ApJ, 781, 87

Bastien, P., \& Menard, F. 1990, ApJ, 364, 232

Benisty, M., Stolker, T., Pohl, A., et al. 2017, A\&A, 597, A42

Beuzit, J.-L., Feldt, M., Dohlen, K., et al. 2008, in Ground-based and Airborne Instrumentation for Astronomy II, Proc. SPIE, 7014, 701418

Boccaletti, A., Abe, L., Baudrand, J., et al. 2008, in Adaptive Optics Systems, Proc. SPIE, 7015, 70151B

Bohren, C. F., \& Huffman, D. R. 1983, Absorption and scattering of light by small particles (New York: Wiley)

Brown, J. M., Blake, G. A., Dullemond, C. P., et al. 2007, ApJ, 664, L107

Canovas, H., Rodenhuis, M., Jeffers, S. V., Min, M., \& Keller, C. U. 2011, A\&A, 531, A102

Canovas, H., Ménard, F., de Boer, J., et al. 2015, A\&A, 582, L7 
Chauvin, G., Lagrange, A.-M., Bonavita, M., et al. 2010, A\&A, 509, A52

Cheetham, A., Huélamo, N., Lacour, S., de Gregorio-Monsalvo, I., \& Tuthill, P. 2015, MNRAS, 450, L1

Cieza, L. A., Olofsson, J., Harvey, P. M., et al. 2011, ApJ, 741, L25

Claudi, R. U., Turatto, M., Gratton, R. G., et al. 2008, in Ground-based and Airborne Instrumentation for Astronomy II, Proc. SPIE, 7014, 70143E

Crida, A., Morbidelli, A., \& Masset, F. 2006, Icarus, 181, 587

Dohlen, K., Langlois, M., Saisse, M., et al. 2008, in Ground-based and Airborne Instrumentation for Astronomy II, Proc. SPIE, 7014, 70143L

Dominik, C., \& Tielens, A. G. G. M. 1997, ApJ, 480, 647

Dong, R., Zhu, Z., \& Whitney, B. 2015, ApJ, 809, 93

Dong, R., Fung, J., \& Chiang, E. 2016, ApJ, 826, 75

Draine, B. T. 2003, ApJ, 598, 1026

Dullemond, C. P., \& Dominik, C. 2005, A\&A, 434, 971

Dullemond, C. P., Juhasz, A., Pohl, A., et al. 2012, Astrophysics Source Code Library [record ascl: 1202.015]

Fischer, O., Henning, T., \& Yorke, H. W. 1996, A\&A, 308, 863

Flock, M., Ruge, J. P., Dzyurkevich, N., et al. 2015, A\&A, 574, A68

Follette, K. B., Grady, C. A., Swearingen, J. R., et al. 2015, ApJ, 798, 132

Fusco, T., Rousset, G., Rabaud, D., et al. 2004, J. Opt. A: Pure and Applied Optics, 6, 585

Fusco, T., Petit, C., Rousset, G., et al. 2006, in Proc. SPIE, 6272, 62720K

Fusco, T., Sauvage, J.-F., Petit, C., et al. 2014, in Adaptive Optics Systems IV, Proc. SPIE, 9148, 91481U

Gaia Collaboration (Brown, A. G. A., et al.) 2016, A\&A, 595, A2

Galicher, R., \& Marois, C. 2011, in Second International Conference on Adaptive Optics for Extremely Large Telescopes. Online at http:// ao4elt2.lesia.obspm.fr

Galicher, R., Marois, C., Macintosh, B., et al. 2016, A\&A, 594, A63

Garufi, A., Quanz, S. P., Schmid, H. M., et al. 2016, A\&A, 588, A8

Ginski, C., Stolker, T., Pinilla, P., et al. 2016, A\&A, 595, A112

Hashimoto, J., Dong, R., Kudo, T., et al. 2012, ApJ, 758, L19

Huélamo, N., Lacour, S., Tuthill, P., et al. 2011, A\&A, 528, L7

Huélamo, N., de Gregorio-Monsalvo, I., Macias, E., et al. 2015, A\&A, 575, L5

Hughes, A. M., Wilner, D. J., Qi, C., \& Hogerheijde, M. R. 2008, ApJ, 678, 1119

Isella, A., Carpenter, J. M., \& Sargent, A. I. 2010a, ApJ, 714, 1746

Isella, A., Natta, A., Wilner, D., Carpenter, J. M., \& Testi, L. 2010b, ApJ, 725, 1735

Janson, M., Thalmann, C., Boccaletti, A., et al. 2016, ApJ, 816, L1

Juhász, A., Benisty, M., Pohl, A., et al. 2015, MNRAS, 451, 1147

Kataoka, A., Okuzumi, S., Tanaka, H., \& Nomura, H. 2014, A\&A, 568, A42

Kataoka, A., Muto, T., Momose, M., et al. 2015, ApJ, 809, 78

Langlois, M., Dohlen, K., Vigan, A., et al. 2014, in Ground-based and Airborne Instrumentation for Astronomy V, Proc. SPIE, 9147, 91471R

Lin, D. N. C., \& Papaloizou, J. 1979, MNRAS, 186, 799

Maire, A.-L., Langlois, M., Dohlen, K., et al. 2016, in Proc. SPIE, 9908, 990834

Marino, S., Perez, S., \& Casassus, S. 2015, ApJ, 798, L44

Marois, C., Lafrenière, D., Doyon, R., Macintosh, B., \& Nadeau, D. 2006, ApJ, 641, 556

Marois, C., Zuckerman, B., Konopacky, Q. M., Macintosh, B., \& Barman, T. 2010, Nature, 468, 1080

Merín, B., Brown, J. M., Oliveira, I., et al. 2010, ApJ, 718, 1200

Mesa, D., Gratton, R., Zurlo, A., et al. 2015, A\&A, 576, A121

Min, M., Canovas, H., Mulders, G. D., \& Keller, C. U. 2012, A\&A, 537, A75

Murphy, S. J., Lawson, W. A., \& Bessell, M. S. 2013, MNRAS, 435, 1325

Ohta, Y., Fukagawa, M., Sitko, M. L., et al. 2016, PASJ, 68, 53

Olofsson, J., Benisty, M., Augereau, J.-C., et al. 2011, A\&A, 528, L6

Olofsson, J., Benisty, M., Le Bouquin, J.-B., et al. 2013, A\&A, 552, A4

Ossenkopf, V. 1993, A\&A, 280, 617

Pavlov, A., Möller-Nilsson, O., Feldt, M., et al. 2008, in Advanced Software and Control for Astronomy II, Proc. SPIE, 7019, 701939

Pinilla, P., Birnstiel, T., Ricci, L., et al. 2012, A\&A, 538, A114

Pinilla, P., de Juan Ovelar, M., Ataiee, S., et al. 2015, A\&A, 573, A9

Pinte, C., Harries, T. J., Min, M., et al. 2009, A\&A, 498, 967

Pohl, A., Pinilla, P., Benisty, M., et al. 2015, MNRAS, 453, 1768

Racine, R., Walker, G. A. H., Nadeau, D., Doyon, R., \& Marois, C. 1999, PASP, 111, 587

Ricci, L., Testi, L., Natta, A., et al. 2010, A\&A, 512, A15

Rice, W. K. M., Wood, K., Armitage, P. J., Whitney, B. A., \& Bjorkman, J. E. 2003, MNRAS, 342, 79

Sallum, S., Eisner, J. A., Close, L. M., et al. 2015, ApJ, 801, 85

Schmid, H. M., Joos, F., \& Tschan, D. 2006, A\&A, 452, 657

Schmid, H.-M., Downing, M., Roelfsema, R., et al. 2012, in Ground-based and Airborne Instrumentation for Astronomy IV, Proc. SPIE, 8446, 84468Y
Shakura, N. I., \& Sunyaev, R. A. 1973, A\&A, 24, 337

Soummer, R. 2005, ApJ, 618, L161

Soummer, R., Pueyo, L., \& Larkin, J. 2012, ApJ, 755, L28

Stolker, T., Dominik, C., Avenhaus, H., et al. 2016, A\&A, 595, A113

Takami, M., Karr, J. L., Hashimoto, J., et al. 2013, ApJ, 772, 145

Tazaki, R., Tanaka, H., Okuzumi, S., Kataoka, A., \& Nomura, H. 2016, ApJ, 823, 70

Thalmann, C., Schmid, H. M., Boccaletti, A., et al. 2008, in Ground-based and Airborne Instrumentation for Astronomy II, Proc. SPIE, 7014, 70143F

Thalmann, C., Grady, C. A., Goto, M., et al. 2010, ApJ, 718, L87

Thalmann, C., Mulders, G. D., Janson, M., et al. 2015, ApJ, 808, L41

Torres, C. A. O., Quast, G. R., Melo, C. H. F., \& Sterzik, M. F. 2008, Young Nearby Loose Associations, ed. B. Reipurth, 757

van der Marel, N., van Dishoeck, E. F., Bruderer, S., Pérez, L., \& Isella, A. 2015, A\&A, 579, A106

van der Marel, N., Verhaar, B. W., van Terwisga, S., et al. 2016, A\&A, 592, A126

Vigan, A., Moutou, C., Langlois, M., et al. 2010, MNRAS, 407, 71

Vigan, A., Bonnefoy, M., Ginski, C., et al. 2016, A\&A, 587, A55

Warren, S. G., \& Brandt, R. E. 2008, J. Geophys. Res. (Atmospheres), 113, D14220

Zubko, V. G., Mennella, V., Colangeli, L., \& Bussoletti, E. 1996, MNRAS, 282, 1321

Zurlo, A., Vigan, A., Mesa, D., et al. 2014, A\&A, 572, A85

1 Max Planck Institute for Astronomy, Königstuhl 17, 69117 Heidelberg, Germany

2 Heidelberg University, Institute of Theoretical Astrophysics, AlbertUeberle-Str. 2, 69120 Heidelberg, Germany

3 INAF-Osservatorio Astronomico di Padova, Vicolo dell'Osservatorio 5, 35122 Padova, Italy

4 Dipartimento di Fisica e Astronomia "G. Galilei”, Universita' degli Studi di Padova, Vicolo dell'Osservatorio 3, 35122 Padova, Italy

5 CRAL, UMR 5574, CNRS, Université Lyon 1, 9 avenue Charles André, 69561 Saint Genis Laval Cedex, France

6 Aix Marseille Université, CNRS, LAM (Laboratoire d'Astrophysique de Marseille) UMR 7326, 13388 Marseille, France

7 European Southern Observatory, Alonso de Córdova 3107, Casilla 19001 Vitacura, Santiago 19, Chile

8 Leiden Observatory, Leiden University, P.O. Box 9513, 2300 RA Leiden, The Netherlands

9 Anton Pannekoek Institute for Astronomy, University of Amsterdam, Science Park 904, 1098 XH Amsterdam, The Netherlands

10 Instituto de Física y Astronomía, Facultad de Ciencias, Universidad de Valparaíso, Av. Gran Bretaña 1111, Playa Ancha, Valparaíso, Chile

11 ICM nucleus on protoplanetary disks, Protoplanetary discs in ALMA Early Science, Universidad de Valparaíso, Valparaíso, Chile

12 Univ. Grenoble Alpes, CNRS, IPAG, 38000 Grenoble, France

13 Institute for Astronomy, The University of Edinburgh, Royal Observatory, Blackford Hill View, Edinburgh, EH9 3HJ, UK

14 LESIA, Observatoire de Paris, PSL Research University, CNRS, Sorbonne Universités, UPMC Univ. Paris 06, Univ. Paris Diderot, Sorbonne Paris Cité, 5 place Jules Janssen, 92195 Meudon, France

15 Institute for Astronomy, ETH Zurich, Wolfgang-Pauli-Strasse 27, 8093 Zurich, Switzerland

16 Department of Astronomy, Stockholm University, AlbaNova University Center, 10691 Stockholm, Sweden

17 Institute of Astronomy, University of Cambridge, Madingley Road, Cambridge CB3 0HA, UK

18 Núcleo de Astronomía, Facultad de Ingeniería, Universidad Diego Portales, Av. Ejercito 441, Santiago, Chile

19 Departamento de Astronomía, Universidad de Chile, Casilla 36-D, Santiago, Chile

20 Observatoire Astronomique de l'Université de Genève, $51 \mathrm{Ch}$. des Maillettes, 1290 Versoix, Switzerland

21 European Southern Observatory, Karl-Schwarzschild-Str. 2, 85748 Garching, Germany 
Appendix A: Synthetic $Q_{\phi}$ image of model \#1
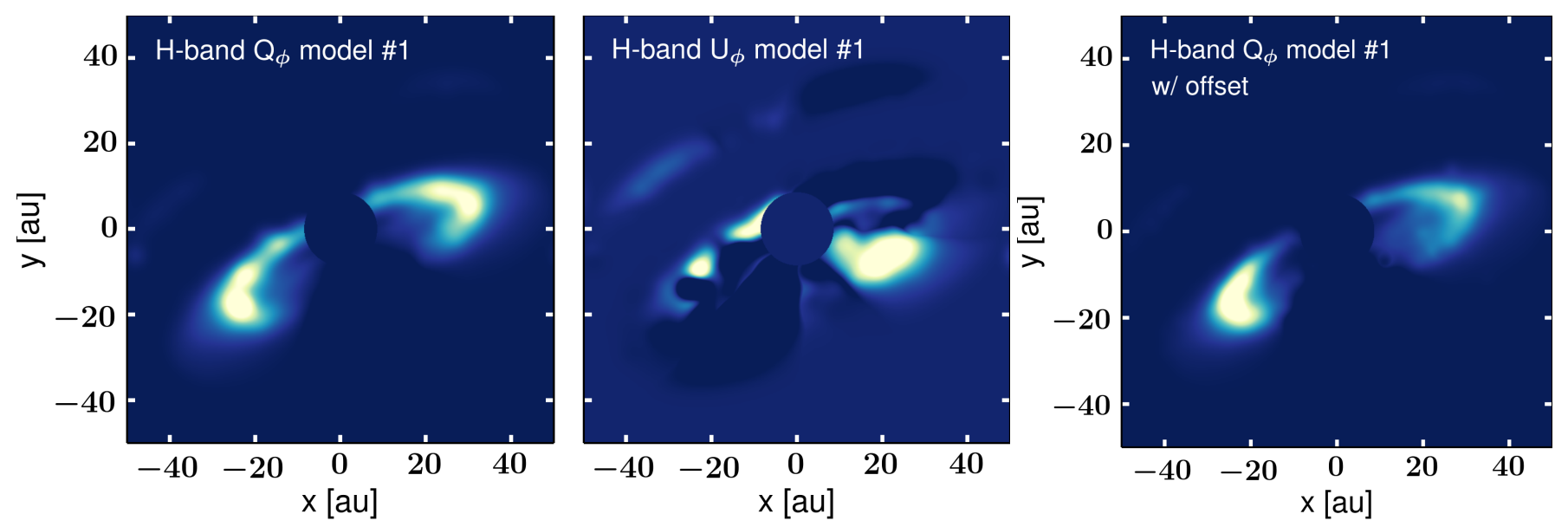

Fig. A.1. $Q_{\phi}$ (left) and $U_{\phi}$ (middle) images of model \#1 at $H$-band. The right panel considers the same model, but the star is slightly offset from its original central position along the semi-major axis. The images are convolved with a Gaussian PSF with FWHM of 0’.04 (at 107 pc distance). The color scale considers the same dynamical range as for the previous synthetic images. 